\title{
1 Pluripotency and the origin of animal multicellularity
}

2

3 Shunsuke Sogabe*1†, William L. Hatleberg*1†, Kevin M. Kocot², Tahsha E. Say', Daniel

4 Stoupin $^{1 \dagger}$, Kathrein E. Roper ${ }^{1 \dagger}$, Selene L. Fernandez-Valverde ${ }^{1 \dagger}$, Sandie M. Degnan ${ }^{1 \#}$ and

5 Bernard M. Degnan ${ }^{1 \#}$

6

7 1. School of Biological Sciences, University of Queensland, Brisbane QLD 4072, Australia

8 2. Department of Biological Sciences and Alabama Museum of Natural History, The

9 University of Alabama, Tuscaloosa, AL 35487 USA

10

$11 *$ These authors contributed equally to this work

12 \# Corresponding authors

13

14 †Present addresses: The Scottish Oceans Institute, Gatty Marine Laboratory, School of

15 Biology, University of St Andrews, East Sands, St Andrews, Fife KY16 8LB, UK (S.S.);

16 Department of Biological Sciences, Carnegie Mellon University, 4400 Fifth Avenue,

17 Pittsburgh, PA 15213 USA (W.L.H.); BioQuest Studios, PO Box 603, Port Douglas

18 QLD 4877, Australia (D.S.); Centre for Clinical Research, Faculty of Medicine, University

19 of Queensland, Herston QLD 4029, Australia (K.R.); CONACYT, Unidad de Genómica

20 Avanzada, Laboratorio Nacional de Genómica para la Biodiversidad, Centro de

21 Investigación y de Estudios Avanzados del IPN, Irapuato, Guanajuato, Mexico (S.L.F.-V.). 
22 The most widely held, but rarely tested, hypothesis for the origin of animals is

23 that they evolved from a unicellular ancestor with an apical cilium surrounded by

a microvillar collar that structurally resembled present-day sponge choanocytes and choanoflagellates ${ }^{1-4}$. Here we test this traditional view of the origin of the animal kingdom by comparing the transcriptomes, fates and behaviours of the three primary sponge cell types - choanocytes, pluripotent mesenchymal archeocytes and epithelial pinacocytes - with choanoflagellates and other unicellular holozoans. Unexpectedly, we find the transcriptome of sponge choanocytes is the least similar to the transcriptomes of choanoflagellates and is significantly enriched in genes unique to either animals or to sponges alone. In contrast, pluripotent archeocytes upregulate genes controlling cell proliferation and gene expression, as in other metazoan stem cells and in the proliferating stages of two closely-related unicellular holozoans, including a colonial choanoflagellate. In the context of the body plan of the sponge, Amphimedon queenslandica, we show that choanocytes appear late in development and are the result of a transdifferentiation event. They exist in a metastable state and readily transdifferentiate into archeocytes, which can differentiate into a range of other cell types. These sponge cell type conversions are similar to the temporal cell state changes that occur in many unicellular holozoans 5 . Together, these analyses offer no support for the homology of sponge choanocytes and choanoflagellates, 42 nor for the view that the first multicellular animals were simple balls of cells with

43 limited capacity to differentiate. Instead, our results are consistent with the first

44 animal cell being able to transition between multiple states in a manner similar to modern transdifferentiating and stem cells. 


\section{Main}

47 The last common ancestor of all living animals appears to have possessed epithelial and

48 mesenchymal cell types that could transdifferentiate over an ontogenetic life cycle

49 (Fig.1a) ${ }^{1,4}$. This capacity to develop and differentiate required a regulatory capacity to

50 control spatial and temporal gene expression, and included a diversified set of signalling

51 pathways, transcription factors, enhancers, promoters and non-coding RNAs (Fig. 1a) ${ }^{5-9}$.

52 Recent analyses of the genomes and life cycles of unicellular holozoan relatives of

53 animals have revealed that the regulatory repertoire present in multicellular animals

54 largely evolved first in a unicellular ancestor (Fig. 1a) ${ }^{2,5,6}$. These insights contrast with a

55 widely-held view that all animals evolved from a stem organism that was a simple ball

56 of ciliated cells ${ }^{1,3,4}$. Implicit in this traditional perspective is that (i) regulatory systems

57 necessary for cell differentiation evolved after the divergence of metazoan and

58 choanoflagellates lineages, and (ii) morphological features shared between

59 choanoflagellate and choanocytes are homologous and were present in the original

60 animal cell. While the former is not supported by recent data - unicellular holozoans

61 can change cell states by environmentally-induced temporal shifts in gene expression

62 (Fig. 1a) $5,6,10-12$ - the latter is contingent upon the still controversial aspect of whether

63 extant choanocytes and choanoflagellates accurately reflect the ancestral animal cell

64 type.

65 To test this, we first compared cell type-specific transcriptomes ${ }^{13}$ from the sponge

66 Amphimedon queenslandica with each other, and with transcriptomes expressed during

67 the life cycles of closely-related unicellular holozoans, the choanoflagellate Salpingoeca

68 rosetta, the filasterean Capsaspora owczarzaki and the ichthyosporean Creolimax

69 fragrantissima (Fig. 1a) ${ }^{10-12}$. We chose three sponge somatic cell types hypothesised to

70 be homologous to cells present in the last common ancestor of contemporary 
71 metazoans, choanozoans or holozoans: (i) choanocytes, which are internal epithelial

72 feeding cells that capture food by pumping water through the sponge; (ii) epithelial cells

73 called pinacocytes, which line internal canals and the outside of the sponge; and (iii)

74 mesenchymal pluripotent stem cells called archeocytes, which inhabit the middle

75 collagenous layer and have a range of other functions (Fig. 1 and Supplementary Video

76 1) ${ }^{2,14-16}$. These three cell types were manually picked and frozen within 15 minutes of $A$.

77 queenslandica being dissociated (see Methods and Supplementary Video 2). Their

78 transcriptomes were sequenced using CEL-Seq2 ${ }^{17}$ and mapped to the Aqu2.1 annotated

79 genome ${ }^{18}$. This approach allowed visual verification of the three cell types, minimised

80 the time for transcriptional changes to occur after cell dissociation, and allowed for

81 deep sequencing of cell type transcriptomes (Extended Data Table 1, and

82 Supplementary Files S1 and S2).

83 Principle component analysis (PCA) and sparse partial least squares discriminant

84 analysis (sPLS-DA) ${ }^{19}$ reveal that the transcriptomes of the three $A$. queenslandica cell

85 types are unique, with choanocytes being the most distinct (Fig. 2a and Extended Data

86 Fig. 1). Of 44,719 protein-coding genes, 11,013 genes were identified as significantly

87 differentially expressed in at least one cell type from pairwise comparisons between the

88 three cell types using DESeq2 ${ }^{20}$ (Fig. 2b and Supplementary File S3). Significant

89 differences between cell types were independently corroborated by sPLS-DA, which

90 highlighted a subset of 110 genes that explain $15 \%$ of the variance in the dataset and

91 clearly discriminate the choanocytes from the other two cell types (Extended Data Fig.

92 1). This subset includes numerous putative immunity genes that typically encode

93 multiple domains in unique configurations, including scavenger receptor cysteine-rich,

94 tetratricopeptide repeat and epidermal growth factor domains (Supplementary File S4). 
95 From the DESeq2 analysis, we find that archeocytes significantly upregulate genes

involved in the control of cell proliferation, transcription and translation, consistent

97 with their function as pluripotent stem cells (Fig. 2c and Supplementary File S5). In

contrast, choanocyte and pinacocyte transcriptomes are enriched in suites of genes

99 involved in cell adhesion, signalling and polarity, consistent with their role as epithelial

100 cells (Fig. 2d; Extended Data Figure 2 and Supplementary File S5).

101 We identified the evolutionary age of all protein coding genes in the Amphimedon

102 genome as well as the genes significantly and uniquely up-regulated in each cell-type

103 specific transcriptome using phylostratigraphy, which is based on sequence similarity

104 with genes in other organisms with a defined phylogenetic distance ${ }^{21}$. Specifically, we

105 classified Amphimedon genes as having evolved (i) before or (ii) after divergence of

106 metazoan and choanoflagellate lineages (these are called pre-metazoan and metazoan

107 genes, respectively), or (iii) after divergence of the sponge lineage from all other

108 animals (sponge-specific genes). In total, the $A$. queenslandica genome is comprised of

$10928 \%$ pre-metazoan, 26\% metazoan and 46\% sponge-specific protein-coding genes (Fig.

$1103 a$ and Supplementary File S6). We find that 43\% of genes significantly up-regulated in

111 choanocytes have homologues detectable only in sponges, which is similar to the entire

112 genome (Fig. 3b). In contrast, $62 \%$ of genes significantly up-regulated in the pluripotent

113 archeocytes belong to the evolutionarily oldest pre-metazoan category, which is

114 significantly higher than $28 \%$ for the entire genome (Fig. 3c). As with archeocytes,

115 pinacocytes express significantly more pre-metazoan and fewer sponge-specific genes

116 than would be expected from the whole genome profile (Fig. 3d). Results supporting

117 this analysis are obtained when we (i) undertake the same phylostratigraphic analysis

118 of all genes expressed in these cell types, taking also into account relative transcript

119 abundances (Extended Data Fig. 3 and Supplementary File S7), or (ii) classify gene age 
120 using an alternative method to identify of orthogroups (homology cluster containing

121 both orthologues and paralogues) ${ }^{22}$ among unicellular holozoan, yeast and Arabidopsis

122 coding sequences (Extended Data Fig. 4).

123 Comparison of $A$. queenslandica cell-type transcriptomes with stage-specific

124 transcriptomes from the choanoflagellate S. rosetta ${ }^{10}$, the filasterean $C$. owczarzaki $^{11}$ and

125 the ichthyosporean $C$. fragrantissima ${ }^{12}$ reveals that archeocytes have a significantly

126 similar transcriptome to the colonial stage of the choanoflagellate and the multinucleate

127 stage of the ichthyosporean (Fig. 3e). Consistent with this result, the significantly up-

128 regulated genes in the colonial or multinucleate stages of all three unicellular holozoans

129 share the highest proportion of orthogroups with genes significantly up-regulated in

130 archeocytes (Extended Data Fig. 5). In contrast, choanocyte and pinacocyte

131 transcriptomes have no significant similarity to any known unicellular holozoan

132 transcriptome, and share a lower proportion of orthogroups with unicellular holozoans

133 compared to archeocytes (Fig. 3e and Extended Data Fig. 5a).

134 When we compare the 94 differentially up-regulated transcription factor genes in $A$.

135 queenslandica choanocytes, pinacocytes and archeocytes, we find no marked difference

136 in their phylostratigraphic age, suggesting that the gene regulatory networks

137 operational in these cells are of an overall similar evolutionary age (Extended Data Fig.

1386 and Supplementary File S8). We detected 20, 25 and 21 orthologues of the 43

139 evolutionarily-oldest (i.e. pre-metazoan) transcription factor genes expressed in the

140 Amphimedon cells in the genomes of Salpingoeca, Capsaspora and Creolimax

141 respectively, with 9 of these being present in all species (Supplementary File S8).

142 Comparison of the expression profiles of the transcription factor genes shared among

143 these unicellular holozoans and Amphimedon revealed no evidence of a conserved, co-

144 expressed gene regulatory network (Extended Data Fig. 7 and Supplementary File S8). 
145 However, the proto-oncogene $M y c$ and its heterodimeric partner Max are up-regulated

146 in A. queenslandica archeocytes (Extended Data Fig. 6), as observed in other metazoan

147 self-renewing pluripotent stem cells ${ }^{23}$. Myc and Max are present also in

148 choanoflagellates, filastereans and ichthyosporeans, where they heterodimerise and

149 bind to E-boxes just as they do in animals ${ }^{10-12,24}$. Myc is expressed in the proliferative

150 stage of Capsaspora, where it regulates genes associated with ribosome biogenesis and

151 translation ${ }^{6}$. Sponge archeocytes also have enriched expression of genes involved in

152 translation, transcription and DNA replication (Fig. 2c). This suggests that Myc's role in

153 regulating proliferation and differentiation predates its role in bilaterian stem cells and

154 cancer 23,25 , and was likely a cardinal feature of the first metazoan cell.

155 Given that we found no transcriptional support for homology of A. queenslandica

156 choanocytes and choanoflagellates, but did find evidence for pluripotent archeocytes

157 expressing a largely premetazoan transcriptome, we sought to investigate the

158 relationships of these cell types in the context of development and the body plan. In

159 Amphimedon and most other sponges, archeocytes form during embryogenesis to

160 populate the inner cell mass of the larva and are the most prevalent cell type during

161 early metamorphosis ${ }^{15}$. As metamorphosis progresses, these archeocytes differentiate

162 into other cell types that populate the juvenile body plan, including choanocytes and

163 pinacocytes, the former of which can transdifferentiate into other cell types ${ }^{16,26}$. To

164 further understand the stability of choanocytes and the dynamics of

165 transdifferentiation, we selectively labelled choanocytes in 3 day old juvenile $A$.

166 queenslandica with CM-DiI (Fig. 4a) and followed their fate over 24 hours (Fig. 4b).

167 Within 4 hours of labelling, many choanocytes dedifferentiated into archeocytes (Fig.

168 4c, d, Supplementary Video 3); this did not require prior cell division (Extended Data

169 Fig. 8). By as little as two hours later, some of these CM-DiI labelled archeocytes had 
170 differentiated into pinacocytes (Fig. 4e); within 12 hours, we detected multiple labelled

171 cell types (Fig. 4e, f). Together, these results suggest that archeocytes are essential in

172 the development and maintenance of the A. queenslandica body plan, as appears to be

173 the case in other sponges ${ }^{15}$. Unlike archeocytes, choanocytes appear late in

174 development and exist in a metastable state, sometimes lasting only a few hours before

175 dedifferentiating back into archeocytes (Fig. 4g, Extended Data Fig. 8).

176 In conclusion, our analysis of sponge and unicellular holozoan cell transcriptomes,

177 development and behaviour provides no support for the long-standing and widely-held

178 hypothesis that multicellular animals evolved from an ancestor that was an

179 undifferentiated ball of cells resembling extant choanocytes and choanoflagellates ${ }^{1-4}$.

180 This conclusion is corroborated by recent studies that question the homology of

181 choanocytes and choanoflagellates based on cell structure ${ }^{27,28}$. As an alternative, we

182 posit that the ancestral metazoan cell type, regardless of its external character, had the

183 capacity to exist in, and transition between, multiple cell states in a manner similar to

184 modern transdifferentiating and stem cells. Previous analyses of holozoan genomes

185 support this postulate, with some of the genomic foundations of pluripotency being

186 established deep in a unicellular past ${ }^{6,24}$. Genomic innovations unique to metazoans,

187 including the origin and expansion of key signalling pathway and transcription factor

188 families, and regulatory DNA and RNA classes ${ }^{7,9,29}$, may have conferred the ability of this

189 ancestral pluripotent cell to evolve a regulatory system where it could co-exist in

190 multiple states of differentiation, giving rise to the first multicellular animal.

191

192 References (Main Text) 
1931 Cavalier-Smith, T. Origin of animal multicellularity: precursors, causes,

194 consequences - the choanoflagellate/sponge transition, neurogenesis and the

195 Cambrian explosion. Philos. Trans. R. Soc. Lond. B Biol. Sci. 372, 20150476 (2017).

1962 Brunet, T. \& King, N. The origin of animal multicellularity and cell differentiation.

197 Dev. Cell 43, 124-140 (2017).

1983 Arendt, D., Benito-Gutierrez, E., Brunet, T. \& Marlow, H. Gastric pouches and the

199 mucociliary sole: setting the stage for nervous system evolution. Philos. Trans. R.

200 Soc. Lond. B Biol. Sci. 370, 20150286 (2015).

2014 Nielsen, C. Six major steps in animal evolution: are we derived sponge larvae? Evol.

202 Dev. 10, 241-257 (2008).

2035 Sebe-Pedros, A., Degnan, B. M. \& Ruiz-Trillo, I. The origin of Metazoa: a unicellular

204 perspective. Nat. Rev. Genet. 18, 498-512 (2017).

2056 Sebe-Pedros, A. et al. The dynamic regulatory genome of Capsaspora and the origin

206 of animal multicellularity. Cell 165, 1224-1237 (2016).

2077 Gaiti, F. et al. Landscape of histone modifications in a sponge reveals the origin of

208 animal cis-regulatory complexity. eLife 6, e22194 (2017).

2098 Gaiti, F., Calcino, A. D., Tanurdzic, M. \& Degnan, B. M. Origin and evolution of the

210 metazoan non-coding regulatory genome. Dev. Biol. 427, 193-202 (2017).

2119 Babonis, L. S. \& Martindale, M. Q. Phylogenetic evidence for the modular evolution

212 of metazoan signalling pathways. Philos. Trans. R. Soc. Lond. B Biol. Sci. 372,

$21320150477(2016)$.

21410 Fairclough, S. R. et al. Premetazoan genome evolution and the regulation of cell

215 differentiation in the choanoflagellate Salpingoeca rosetta. Genome Biol. 14, R15

$216 \quad$ (2013). 
21711 Sebé-Pedrós, A. et al. Regulated aggregative multicellularity in a close unicellular

218 relative of Metazoa. eLife 2, e01287 (2013).

21912 de Mendoza, A., Suga, H., Permanyer, J., Irimia, M. \& Ruiz-Trillo, I. Complex

220 transcriptional regulation and independent evolution of fungal-like traits in a

221 relative of animals. eLife 4, e08904 (2015).

22213 Arendt, D. et al. The origin and evolution of cell types. Nat. Rev. Genet. 17, 744-757

223 (2016).

22414 Maldonado, M. Choanoflagellates, choanocytes, and animal multicellularity. Invert.

$225 \quad$ Biol. 123, 1-22 (2004).

22615 Ereskovsky, A. The Comparative Embryology of Sponges. Springer, Netherlands

227 (2010).

22816 Nakanishi, N., Sogabe, S. \& Degnan, B. Evolutionary origin of gastrulation: insights

229 from sponge development. BMC Biol. 12, 26 (2014).

23017 Hashimshony, T. et al. CEL-Seq2: sensitive highly-multiplexed single-cell RNA-Seq.

$231 \quad$ Genome Biol. 17, 77 (2016).

23218 Fernandez-Valverde, S. L., Calcino, A. D. \& Degnan, B. M. Deep developmental

233 transcriptome sequencing uncovers numerous new genes and enhances gene

234 annotation in the sponge Amphimedon queenslandica. BMC Genom. 16, 387 (2015).

23519 Le Cao, K. A., Boitard, S. \& Besse, P. Sparse PLS discriminant analysis: biologically

236 relevant feature selection and graphical displays for multiclass problems. $B M C$

237 Bioinform. 12, 253 (2011).

23820 Love, M. I., Huber, W. \& Anders, S. Moderated estimation of fold change and

239 dispersion for RNA-seq data with DESeq2. Genome Biol. 15, 1-21 (2014).

24021 Domazet-Lošo, T. \& Tautz, D. A phylogenetically based transcriptome age index

241 mirrors ontogenetic divergence patterns. Nature 468, 815-818 (2010). 
24222 Li, L., Stoeckert, C. J. \& Roos, D. S. OrthoMCL: identification of ortholog groups for

243 eukaryotic genomes. Genome Res. 13, 2178-2189 (2003).

24423 Fagnocchi, L. \& Zippo, A. Multiple roles of MYC in integrating regulatory networks of

245 pluripotent stem cells. Front. Cell Dev. Biol. 5, 7 (2017).

24624 Young, S. L., Diolaiti, D., Conacci-Sorrell, M., Ruiz-Trillo, I., Eisenman, R. N. \& King, N.

247 Premetazoan ancestry of the Myc-Max network. Mol. Biol. Evol. 28, 2961-2971

$248 \quad$ (2011).

24925 Kress, T. R., Sabo, A. \& Amati, B. MYC: connecting selective transcriptional control to 250 global RNA production. Nat. Rev. Cancer 15, 593-607 (2015).

25126 Sogabe, S., Nakanishi, N. \& Degnan, B. M. The ontogeny of choanocyte chambers

252 during metamorphosis in the demosponge Amphimedon queenslandica. EvoDevo 7,

$253 \quad 6(2016)$.

25427 Mah, J. L., Christensen-Dalsgaard, K. K., \& Leys, S. P. Choanoflagellate and

255 choanocyte collar-flagellar systems and the assumption of homology. Evol. Dev. 16,

$256 \quad 25-37(2014)$.

25728 Pozdnyakov, I., Sokolova, A., Ereskovsky, A., \& Karpov, S. Kinetid structure of

258 choanoflagellates and choanocytes of sponges does not support their close

259 relationship. Protistology 11, 248-264 (2017).

26029 Srivastava, M. et al. The Amphimedon queenslandica genome and the evolution of

261 animal complexity. Nature 466, 720-726 (2010).

262

263 Supplementary Information is linked to the online version of the paper at

264 www.nature.com/nature. (This submission includes eight Supplementary Information

265 data files as well as additional material that is available on Dryad.) 


\section{Acknowledgements}

268 This study was supported by funds from the Australian Research Council (B.M.D. and

269 S.M.D.). We thank Iñaki Ruiz Trillo for primary expression data for Capsaspora and

270 Creolimax.

271

\section{Author Contributions}

273 B.M.D and S.M.D conceived and designed the project. S.S., D.S. and K.R. identified and

274 isolated the cells, and prepared the libraries. W.H., S.S and K.M.K. undertook gene

275 expression and annotation, and phylostratigraphic analyses with help from T.S., S.M.D,

276 S. F.-V and B.M.D. S.S. undertook cell lineage analyses. B.M.D, S.M.D and S.S. wrote the

277 manuscript with comments and contributions from all authors.

278

279 Author Information

280 Data deposition statement

281 Amphimedon queenslandica genome sequence can be accessed at

282 (http://metazoa.ensembl.org/Amphimedon queenslandica/Info/Index).

283 All cell-type transcriptome data are available in the NCBI SRA database under the

284 BioProject PRJNA412708. Additional supplementary data is available upon request

285

286

287

288

289

\section{Competing financial interests}

291 The authors declare no competing financial interests. 
293 Corresponding author

294 Correspondence and requests for materials should be addressed to

295 b.degnan@uq.edu.au or s.degnan@uq.edu.au.

297 Figures Legends

299 Figure 1. Holozoan relationships and sponge cell types.

300 a, Cellular and regulatory traits of metazoans and closely related unicellular holozoans.

301 Black dots, trait present; white dots, trait absent; grey dots, trait present but to a lesser

302 extent. Two major evolutionary events are mapped onto the holozoan phylogenetic

303 tree: (i) environmentally-induced, facultative changes in cell state ancestral to

304 holozoans; and (ii) obligate metazoan multicellularity. b, Whole mount internal view of

305 a juvenile Amphimedon queenslandica. Cell types are outlined. A, archeocyte (cluster of

306 four outlined); Cc, choanocyte chamber; S, sclerocyte; Sp, spherulous cell; P, pinacocyte.

307 c, Choanocyte chamber labelled with DiI with an illustration of a single choanocyte

308 below. d, Pinacocyte labelled with DiI with illustration below. e, Archeocyte labelled

309 with DiI with illustration below. Scale bars: b, $10 \mu \mathrm{m}$; c-e, $5 \mu \mathrm{m}$.

311 Figure 2. Comparison of choanocyte, archeocyte and pinacocyte transcriptomes.

312 a, PCA plot of CEL-Seq2 transcriptomes with 95\% confidence level ellipse plots. Blue,

313 choanocytes; red, archeocytes; green, pinacocytes. b, Venn diagram summary of the

314 number of significantly up-regulated genes based on pairwise comparisons between

315 each of the three cell types using DESeq2 with a false discovery rate (FDR) $<0.05$. The

316 percentages are of the total genes differentially up-regulated in all cell types. c, 
317 Percentage of KEGG Genetic Information Processing genes present in each cell type,

318 corresponding to the number of components making up each KEGG category identified.

319 d, Heat map of the expression of Amphimedon epithelial cell polarity, junction and basal

320 lamina genes in each cell type.

321

322 Figure 3. Analysis of gene age of choanocyte, archeocyte and pinacocyte

323 transcriptomes.

324 a, Phylostratigraphic estimate of the evolutionary age of coding genes in the $A$.

325 queenslandica genome. b-d, Estimate of gene age of differentially-expressed genes in

326 choanocytes (b), archeocytes (c) and pinacocytes (d) and the enrichment of phylostrata

327 relative to the whole genome (bottom). Asterisks indicate significant difference (p-value

$328<0.001)$ from the whole genome. e, A heat map comparing orthologous genes uniquely

329 up-regulated in A. queenslandica cell types, and Salpingoeca rosetta, Capsaspora

330 owczarzaki and Creolimax fragrantissima life cycle stages. Colour indicates the

331 significance of overlap based on the odds ratio. Values indicate adjusted p-values and

332 show significant resemblance only between the archeocyte and the $S$. rosetta colonial

333 stage and the $C$. fragrantissima multinucleate stage. N.s., not significant.

Figure 4. Transdifferentiation of choanocytes in Amphimedon queenslandica.

$336 \mathbf{a}, \mathbf{b}$, Whole mount views of 4 day old juveniles labelled with CM-DiI. a, 30 min after CM-

337 DiI labelling. Labelling is almost exclusively in choanocytes in chambers; insert, a single

338 labelled choanocyte chamber. b, 24 hours after labelling. CM-DiI labelling spread

339 throughout the juvenile with limited staining still present in choanocyte chambers;

340 insert, a choanocyte chamber comprising largely of unlabelled cells. c, d, 2 hours (c) and

3414 hours (d) after labelling. Labelled cells (arrow) migrating outside of choanocyte 
342 chambers (dotted lines), some of which have a large nucleus and a clearly visible

343 nucleolus (arrowheads) characteristic of archeocytes. e, 6 hours after initial DiI

344 labelling of choanocytes, labelled pinacocytes (arrow) with thin pseudopodia are

345 detected. f, 12 hours after initial labelling, CM-DiI labelled skeletal sclerocytes (arrow)

346 and other cell types are present. g, Summary diagram of cell type transition in the $A$.

347 queenslandica juvenile. Scale bars: a, b, $200 \mu \mathrm{m}$; c-f, $10 \mu \mathrm{m}$.

Methods

351

\section{Cell isolation}

353 Adult Amphimedon queenslandica were collected from Heron Island Reef, Great Barrier

354 Reef and transferred to a closed aquarium facility where they were housed for no more

355 than three days before being cut into approximately $1 \mathrm{~cm}^{3}$ cubes. These cubes were

356 mechanically dissociated by squeezing through a $20 \mu \mathrm{m}$ mesh. The resultant cell

357 suspension was diluted with $0.22 \mu \mathrm{m}$-filtered seawater (FSW) and the target cell types

358 were identified microscopically based on morphology. Archeocytes are much larger

359 than the other cells and possess a highly visible nucleolus. Choanocytes remain in intact

360 choanocyte chambers after dissociation. Pinacocytes, unlike the other cell types, are

361 translucent and maintain protruding cytoplasmic processes after dissociation. This

362 approach avoided misidentification of dissociated cell types, but could not determine

363 whether these cells are in the process of dividing or differentiating. Individual cells or

364 choanocyte chambers were collected under an inverted microscope (Nikon Eclipse Ti

365 microscope) using a micropipette mounted on micromanipulator (MN-4, Narishige)

366 connected to CellTram Oil (Eppendorf) (Supplementary video 2), flash frozen and 
367 stored at $-80^{\circ} \mathrm{C}$. All cells were frozen within 15 min of dissociation. Samples used in CEL-

368 Seq2 were comprised of pools of either five to six archeocytes or pinacocytes, or a single

369 choanocyte chamber ( 40-60 cells) (Extended Data Table 1$)$. Based on differences in

370 cell size, we estimated that these pools have similar amounts of total RNA. Three

371 pinacocyte, and five archeocyte and choanocyte samples were collected from each of

372 three sponges (Supplementary File S2).

373

374 CEL-Seq2 sample preparation, sequencing and analysis

375 Samples were prepared according to the CEL-Seq2 protocol ${ }^{17}$ and sequenced on two

376 lanes of Illumina HiSeq2500 on rapid mode using HiSeq Rapid SBS v2 reagents

377 (Illumina); CEL-Seq2 libraries were randomised in relation to cell type and source adult

378 sponge in these two lanes. CEL-Seq2 reads were processed using a publicly available

379 pipeline (https://github.com/yanailab/CEL-Seq-pipeline; see additional supplementary

380 data on Dryad: /CEL-Seq pipeline/). Read counts were obtained from demultiplexed

381 reads mapped to A. queenslandica Aqu2.1 gene models ${ }^{18}$. Samples with read counts less

382 than $10^{6}$ were removed and not included in subsequent analyses (Supplementary File

383 S2). For the samples included in the final analysis, approximately $60 \%$ of the reads

384 successfully mapped to the genome (Extended Data Table 1), as per other studies using

385 CEL-Seq ${ }^{30}$.

387 Analysis of differentially expressed genes

388 The mapped read counts were analysed for differential gene expression using the

389 bioconductor package DESeq2 $2^{20,31}$ (see additional supplementary data on Dryad:

$390 / \mathrm{DESeq} 2 /$ ). Genes that had read counts with a row sum of zero were removed. Principle

391 component analyses (PCA) were performed on blind variance stabilising transformed 
392 (vst) counts obtained using DESeq2 and were visualised using the ggplot2 package ${ }^{32}$.

393 Pairwise comparisons were conducted between each of the three cell types to generate

394 a differentially expressed gene (DEG) list for each cell type using a false discovery rate

395 (FDR) $<0.05$. Venn diagrams were generated using VENNY

396 (http://bioinfogp.cnb.csic.es/tools/venny) to visualise and compare the list of DEGs

397 between each cell type. Heat maps were generated using the R-packages pheatmap 33

398 and RColorBrewer ${ }^{34}$ to visualise the expression patterns between the cell types using

399 the vst transformed counts, which were scaled into $\mathrm{z}$ score values ranging from -1 (low

400 expression) to 1 (high expression).

401 All protein coding genes were annotated using blastp (e-value cutoff $=1 e-3$ ) and

402 InterProScan (default settings), which were merged in Blast2G035,36. KEGG annotations

403 were obtained using the online tool BlastKOALA ${ }^{37}$ (see additional supplementary data

404 on Dryad: /KEGG annotation). Pathway analyses were performed using the annotations

405 on the KEGG Mapper - Reconstruct Pathway tool ${ }^{38}$. Complete DEG lists with BLAST2GO,

406 InterPro, Pfam, and phylostrata ID can be found in Supplementary File S3, as well as

407 KEGG pathway enrichments in Supplementary File S5.

408 To identify the genes that best explain differences among cell type transcriptomes,

409 we adopted the multivariate sparse Partial Least Squares Discriminant Analysis (sPLS-

410 DA) ${ }^{19}$, implemented in the mixOmics package ${ }^{39}$ in R v3.3.1 (see additional

411 supplementary data on Dryad: /sPLS-DA/README.txt). This is a supervised analysis

412 that uses the sample information (cell type) to identify the most predictive genes for

413 classifying the samples according to cell type. The optimised numbers of genes per

414 component were obtained by training and correctly evaluating the performance of the

415 predictive model using 5-fold cross-validation, repeated 100 times. A sample plot was

416 used to visualise the similarities between samples for the final sPLS-DA model with 
$41795 \%$ confidence ellipses using the plotIndiv function in R. A heat map was used to

418 visualise relative expression levels of the selected gene models for the two components,

419 using vst counts and the package pheatmap ${ }^{33}$ in R. Venn diagrams were generated using

420 VENNY to visualise and compare the DEGs generated by DESeq2 and sPLS-DA.

421

422 Phylostratigraphy

423 To estimate the evolutionary age of genes up-regulated in each cell type,

424 phylostratigraphy analyses ${ }^{21}$ were performed using blastp and an e-value cutoff of

4250.001 on a custom database containing 1,757 genomes and transcriptomes ${ }^{40}$ that was

426 modified to account for A. queenslandica's phylogenetic position (i.e. all eumetazoan and

427 bilaterian taxa were moved into the metazoan phylostratum, and three phylostrata -

428 poriferan, demosponge and haplosclerid - were added to increase the representation of

429 poriferan transcriptomes; Supplementary File S6, see additional supplementary data on

430 Dryad: /Phylostratigraphy annotations/). Every gene model in A. queenslandica was

431 blasted against each sequence in the database, and its age of gene origin was inferred

432 based on the oldest blast hit relative to a predetermined phylogenetic tree ( $\underline{\text { see }}$

433 additional supplementary data on Dryad: /Phylostratigraphy annotations/).

434 Phylostrata enrichments were performed using the Fisher's exact test ${ }^{41}$ in the

435 BioConductor package, GeneOverlap ${ }^{42}$ in R, to identify significant differences in gene

436 age of the cell type DEG lists relative to the genome (see additional supplementary data

437 on Dryad: /Fig.3b-d and /ED_Fig3_files). Enrichment (log odds ratio value above 0) and

438 under-representation (log odds ratio value below 0 ) of each phylostrata found in the

439 cell type DEG lists relative to the genome, were visualised using the R-packages

440 pheatmap 33 and RColorBrewer ${ }^{34}$. 


\section{Orthology analyses}

443 Orthology analyses were performed using FastOrtho ${ }^{43}$ from a custom 'all-vs-all' blastp

444 database of coding sequences from the genomes of Saccharomyces cerevisiae ${ }^{44}$,

445 Arabidopsis thaliana ${ }^{45}$, Creolimax fragrantissima ${ }^{12}$, Sphaeroforma arctica ${ }^{46}$, Capsaspora

446 owczarzaki ${ }^{47}$, Monosiga brevicollis ${ }^{48}$, and Salpingoeca rosetta ${ }^{10}$, using the following

447 configuration settings: pv_cutoff $=1 \mathrm{e}-5$; pi_cutoff $=0.0$; pmatch_cutoff $=0.0$;

448 maximum_weight $=316.0$; inflation $=1.5$; blast_e $=1 \mathrm{e}-5$ ( $\underline{\text { see additional supplementary }}$

449 data on Dryad: /FastOrtho/). FastOrtho classifies all of the genes present in each

450 genome into orthology groups (orthogroups, OGs), which contain all orthologous and

451 paralogous genes from each species. Genes that do not have any orthologues in other

452 species or paralogues within the same genome were not included in any orthogroups.

453 To compare the gene lists between species in all downstream analyses, species-specific

454 gene names were changed to the common orthogroup identifier.

455 Orthology analyses between A. queenslandica and S. rosetta, C. fragrantissima, and $C$.

owczarzaki cell types were performed using the cell type-specific DEG lists obtained

457 from previous studies on S. rosetta ${ }^{10}$, C. fragrantissima ${ }^{12}$, and $C$. owczarzaki ${ }^{11}$. The

458 BioConductor package, GeneOverlap ${ }^{42}$, was used to identify (1) the number overlapping

459 OGs between species and cell type, and (2) the statistical significance of that overlap

460 based on list size and total number of OGs (see additional supplementary data on Dryad:

461 /Fig.3e). This function provided the odds ratio between the OG lists, where the null

462 hypothesis was no significant overlap (odds ratio value of 1 or smaller) and the

463 alternative being a significant overlap detected between the lists (odds ratio value over

464 1), as well as a p-value calculated for odds ratio values over 1.

465 To supplement phylostratigraphy analyses of Amphimedon cell-type specific gene

466 lists (Fig. 3 and Extended Data Fig. 3), the BioConductor package, GeneOverlap ${ }^{42}$ was 
467 used to identify the number and percentage of orthogroups that are also present in the

468 genomes of Arabidopsis thaliana, Saccharomyces cerevisiae, Creolimax fragrantissima,

469 Sphaeroforma arctica, Capsaspora owczarzaki, Monosiga brevicollis, and Salpingoeca

470 rosetta (Extended Data Fig. 4 and Extended data Fig. 5; see additional supplementary

471 data on Dryad: /ED_Fig4 and ED_Fig5)

472

473 Classification of gene expression levels into quartiles

474 In addition to differential gene expression analyses for Amphimedon transcriptomes, the

475 relative gene expression levels for all cell types were assigned to one of four expression

476 quartiles based on the number of reads that mapped to a given Aqu2.1 gene model

477 (Extended data Fig. 3). All zero read counts were discarded and the mean expression

478 value of the non-transformed normalised count values of all samples (from all cell

479 types) was used to calculate the quartile values. These values $\left(\mathrm{Q}_{1}: 2.30, \mathrm{Q}_{2}: 6.06, \mathrm{Q}_{3}\right.$ :

480 15.83) were used to classify the expression of all of genes in each cell type into four

481 groups based on transcript abundance, ranging from lowest (Q1) to highest (Q4).

482 Phylostrata enrichments for the different quartile value thresholds were performed

483 as described above for the cell type DEG lists; heat maps were generated using

484 pheatmaps ${ }^{33}$ in R ( see additional supplementary data on Dryad: /ED_Fig3_files). All

485 downstream analyses used the median value $\left(Q_{2}: 6.06\right)$ as a cut-off value to obtain a list

486 of expressed genes. Orthology analyses using FastOrtho were performed as described

487 above, and the percentage of genes with shared orthologous group (OG) in each gene

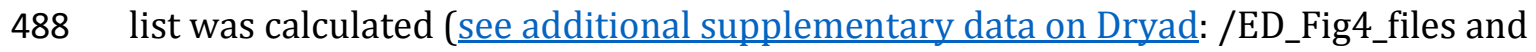

489 ED_Fig5_files). In these analyses, exclusive lists refer to all of the regions in the Venn

490 diagram being treated as a separate list (e.g. archeocyte only, common between

491 archeocyte and choanocyte, common between archeocyte and pinacocyte, etc.), while 
492 non-exclusive lists collapse all of the lists containing a given cell type into one list (e.g.

493 archeocyte non-exclusive DEG list includes, archeocyte DEGs + (archeocyte + pinacocyte

494 DEGs) + (archeocyte + choanocyte DEGs).

495

496 Identification and analysis of expressed $A$. queenslandica transcription factors

497 A list of $A$. queenslandica transcription factors expressed in the three cell types was

498 obtained using a number of independent methods. First, a non-conservative list of

499 putative A. queenslandica transcription factors was obtained using the DNA-binding

500 domain database (DBD: Transcription factor prediction database) and the Pfam IDs of

501 sequence specific DNA-binding domain (DBD) families, which corresponds to known

502 transcription factor families (www.transcriptionfactor.org ${ }^{49}$ ). Second, we collated a list

503 of annotated A. queenslandica transcription factors in the literature $7,16,47,50-66$

504 (Supplementary File S8). Third, we compared these lists to an unpublished in-house

505 database for A. queenslandica (Degnan et al. unpublished) and putative transcription

506 factors identified by OrthoMCL. The final list of 173 expressed transcription factor

507 genes used in this study were present in at least two of the three lists (Supplementary

508 File S8).

509 The evolutionary age of each of the expressed transcription factors was first assigned

510 based on the DBD contained in the gene model and then manually curated based

511 primarily on literature (Supplementary File S8). From this, each TF was assigned as

512 either originating in sponges after diverging form other animals (sponge-specific), in

513 metazoans after they diverged from choanoflagellates (metazoan) or before metazoans

514 diverged from choanoflagellates (premetazoan).

515

516 Analysis of juvenile cell fate and proliferation 
517 Larvae were collected as previously described 67 , left in FSW overnight and then placed

518 in sterile 6-well plates with $10 \mathrm{ml}$ of FSW for 1 hour in the dark with live coralline algae

519 Amphiroa fragilissima. Postlarvae settled on A. fragilissima were removed using fine

520 forceps (Dumont \#5) and resettled on to round coverslips placed in a well with $2 \mathrm{ml}$

521 FSW in a sterile 24-well plastic plate, with 3 postlarvae placed on each coverslip.

522 Metamorphosis from resettled postlarvae to a functional juvenile takes approximately

52372 hours ${ }^{16,68}$. For all samples, FSW was changed daily until fixation.

$524 \quad$ The lipophilic cell tracker CM-DiI (Molecular Probes C7000) was used to label

525 choanocyte chambers in juveniles as previously described ${ }^{16}$, with slight modifications in

526 the concentration used and incubation times. A. queenslandica juveniles were incubated

527 in $1 \mu \mathrm{M}$ CM-DiI in FSW for 30 minutes to 1 hour. This minimised the labelling of non-

528 choanocyte cells. Despite this precaution, some non-choanocyte cells would be labelled

529 in some individuals. Hence, all CM-DiI labelled juveniles were inspected by

530 epifluorescence microscopy (Nikon Eclipse Ti microscope) immediately after CM-DiI

531 was washed out, with juveniles detected with CM-DiI labelled cells outside of

532 choanocyte chambers discarded from the study. Juveniles were allowed to develop for

$5330,2,4,6,12$ or 24 hours post-incubation (hpi) with CM-DiI, then washed in FSW three

534 times for 5 minutes and fixed ${ }^{69}$ without dehydration in ethanol. Fixed juveniles were

535 washed three times in MOPST (1x MOPS buffer $+0.1 \%$ Tween). Nuclei were labelled

536 with DAPI (1:1,000, Molecular Probes) for 30 minutes, washed in MOPST for 5 minutes

537 and mounted using ProlongGold antifade reagent (Molecular Probes). All samples were

538 observed using the ZEISS LSM 710 META confocal microscope, and image analysis was

539 performed using the software ImageJ.

540 To visualise cell proliferation, the thymidine analogue EdU (Click-iT EdU AlexaFluor

541488 cell proliferation kit, Molecular Probes C10337) was used as previously 
542 described ${ }^{16,26}$. To label S-phase nuclei, juveniles were incubated in FSW containing 200

$543 \mu \mathrm{M}$ EdU for 6 hours, washed in FSW and immediately fixed as described above.

544 Fluorescent labelling of incorporated EdU was conducted according to the

545 manufacturer's recommendations prior to DAPI labelling and mounting in ProLong Gold

546 antifade reagent as described above.

547

548

549 References (Methods)

550

55130 Levin, M. et al. The mid-developmental transition and the evolution of animal body

552 plans. Nature 531, 637-641 (2016).

55331 Anders, S. \& Huber, W. Differential expression analysis for sequence count data.

$554 \quad$ Genome Biol. 11, R106 (2010).

55532 Wickham, H. ggplot2: Elegant Graphics for Data Analysis (Springer, 2009).

55633 Kolde, R. Package 'pheatmap'. https://cran.r-project.org/package=pheatmap

$557 \quad$ (2012).

55834 Neuwirth, E. Package 'RColorBrewer'. https://cran.r-

559 project.org/package=RColorBrewer (2011).

56035 Conesa, A. et al. Blast2GO: a universal tool for annotation, visualization and analysis

561 in functional genomics research. Bioinformatics 21, 3674-3676 (2005).

56236 Götz, S. et al. High-throughput functional annotation and data mining with the

563 Blast2G0 suite. Nucleic Acids Res. 36, 3420-3435 (2008).

56437 Kanehisa, M., Sato, Y. \& Morishima, K. BlastKOALA and GhostKOALA: KEGG tools for

565 functional characterization of genome and metagenome sequences. J. Mol. Biol. 428,

$566 \quad 726-731(2016)$ 
56738 Kanehisa, M., Sato, Y., Kawashima, M., Furumichi, M. \& Tanabe, M. KEGG as a

568 reference resource for gene and protein annotation. Nucleic Acids Res. 44, D457-

569 D462 (2015).

57039 Rohart, F., Gautier, B., Singh, A. \& Le Cao, K.-A. mixOmics: an R package for 'omics

571 feature selection and multiple data integration. PLoS Comput. Biol. 13, e1005752

$572 \quad$ (2017).

57340 Aguilera, F., McDougall, C. \& Degnan, B. M. Co-Option and de novo gene evolution

$574 \quad$ underlie molluscan shell diversity. Mol. Biol. Evol. 34, 779-792 (2017).

57541 Domazet-Lošo, T., Brajković, J. \& Tautz, D. A phylostratigraphy approach to uncover

576 the genomic history of major adaptations in metazoan lineages. Trends Genet. 23,

$577 \quad 533-539$ (2007).

57842 Shen, L. GeneOverlap: An R package to test and visualize gene overlaps. (2014).

57943 Wattam, A. R. et al. PATRIC, the bacterial bioinformatics database and analysis

$580 \quad$ resource. Nucleic Acids Res. 42, D581-591 (2014).

58144 Yates, A. et al. Ensembl 2016. Nucleic Acids Res. 44, D710-D716 (2016).

58245 The Arabidopsis Genome Initiative. Analysis of the genome sequence of the

583 flowering plant Arabidopsis thaliana. Nature 408, 796-815 (2000).

58446 Ruiz-Trillo, I., Lane, C. E., Archibald, J. M. \& Roger, A. J. Insights into the evolutionary

585 origin and genome architecture of the unicellular opisthokonts Capsaspora

586 owczarzaki and Sphaeroforma arctica. J. Eukaryot. Microbiol. 53, 379-384 (2006).

58747 Suga, H. et al. The Capsaspora genome reveals a complex unicellular prehistory of

$588 \quad$ animals. Nat. Commun. 4, 2325 (2013).

58948 King, N. et al. The genome of the choanoflagellate Monosiga brevicollis and the origin

$590 \quad$ of metazoans. Nature 451, 783-788 (2008). 
59149 Wilson, D., Charoensawan, V., Kummerfeld, S. K. \& Teichmann, S. A. DBD -

592 taxonomically broad transcription factor predictions: new content and

593 functionality. Nucleic Acids Res. 36, D88-92 (2008).

59450 Babonis, L. S. \& Martindale, M. Q. Phylogenetic evidence for the modular evolution

595 of metazoan signalling pathways. Philos. Trans. R. Soc. Lond. B. Biol. Sci. 372,

$596 \quad 20150477$ (2017).

59751 Srivastava, M. et al. Early evolution of the LIM homeobox gene family. BMC Biol. 8, 4 $598 \quad$ (2010).

59952 Larroux, C. et al. Genesis and expansion of metazoan transcription factor gene $600 \quad$ classes. Mol. Biol. Evol. 25, 980-996 (2008).

60153 Larroux, C. et al. Developmental expression of transcription factor genes in a 602 demosponge: insights into the origin of metazoan multicellularity. Evol. Dev. 8, 150$603 \quad 173(2006)$.

60454 Shimeld, S. M., Degnan, B. \& Luke, G. N. Evolutionary genomics of the Fox genes:

605 origin of gene families and the ancestry of gene clusters. Genomics 95, 256-260 $606 \quad$ (2010).

60755 Layden, M. J., Meyer, N. P., Pang, K., Seaver, E. C. \& Martindale, M. Q. Expression and 608 phylogenetic analysis of the zic gene family in the evolution and development of 609 metazoans. EvoDevo 1, 12 (2010).

61056 Presnell, J. S., Schnitzler, C. E. \& Browne, W. E. KLF/SP transcription factor family 611 evolution: Expansion, diversification, and innovation in eukaryotes. Genome Biol. $612 \quad$ Evol. 7, 2289-2309 (2015).

61357 Mukhopadhyay, S. \& Jackson, P. K. The tubby family proteins. Genome Biol. 12, 225

614 (2011). 
61558 Larroux, C. et al. The NK homeobox gene cluster predates the origin of Hox genes.

$616 \quad$ Curr. Biol. 17, 706-710 (2007).

61759 Wang, L., Tang, Y., Cole, P. A. \& Marmorstein, R. Structure and chemistry of the

618 p300/CBP and Rtt109 histone acetyltransferases: Implications for histone

619 acetyltransferase evolution and function. Curr. Opin. Struct. Biol. 18, 741-747

$620 \quad$ (2008).

62160 Petroni, K. et al. The promiscuous life of plant NUCLEAR FACTOR Y transcription

622 factors. Plant Cell 24, 4777-4792 (2012).

62361 Morrison, A. J. \& Shen, X. Chromatin remodelling beyond transcription: the IN080

624 and SWR1 complexes. Nat. Rev. Mol. Cell Biol. 10, 373-384 (2009).

62562 Jones, M. H., Hamana, N., Nezu, J. \& Shimane, M. A novel family of bromodomain

626 genes. Genomics 63, 40-45 (2000).

62763 Song, W., Solimeo, H., Rupert, R. A., Yadav, N. S. \& Zhu, Q. Functional dissection of a

628 Rice Dr1/DrAp1 transcriptional repression complex. Plant Cell 14, 181-195 (2002).

62964 Matheos, D. P., Kingsbury, T. J., Ahsan, U. S. \& Cunningham, K. W. Tcn1p/Crz1p, a

630 calcineurin-dependent transcription factor that differentially regulates gene

631 expression in Saccharomyces cerevisiae. Genes Dev. 11, 3445-3458 (1997).

63265 Rivera, A. S. et al. Gene duplication and the origins of morphological complexity in

633 pancrustacean eyes, a genomic approach. BMC Evol. Biol. 10, 123, (2010).

63466 Romanovskaya, E. V. et al. Transcription factors of the NF1 family: Possible

635 mechanisms of inducible gene expression in the evolutionary lineage of

636 multicellular animals. J. Evol. Biochem. Physiol. 53, 85-92 (2017).

63767 Leys, S. P. et al. Isolation of Amphimedon developmental material. Cold Spring Harb.

$638 \quad$ Protoc. 3, 5095 (2008). 
63968 Degnan, B. M. et al. Porifera. Evolutionary Developmental Biology of Invertebrates

$640 \quad$ vol.1 (Springer, 2015).

64169 Larroux, C. et al. Whole-mount in situ hybridization in Amphimedon. Cold Spring

$642 \quad$ Harb. Protoc. 3, 5096 (2008).

643

644

645

646 Extended Data Figure Legends

647

648 Extended Data Figure 1: Sparse partial least squares discriminant analysis (sPLS-

649 DA) of Amphimedon queenslandica choanocyte, archeocyte and pinacocyte

650 transcriptomes.

651 A supervised multivariate analysis, sPLS-DA, identified the gene models that best

652 characterise differences in choanocytes (blue), archeocytes (red) and pinacocytes

653 (green). a, Sample plot for the optimal number of gene models that discriminate cell

654 types on the first two components; ellipses indicate $95 \%$ confidence intervals. b, c,

655 Hierarchically-clustered heat maps show the expression of (b) the 110 gene models

656 selected for the first component, and (c) the 98 gene models and 2 long non-coding

657 RNAs selected for the second component, which accounted for $15 \%$ and $5 \%$ of

658 explained variance, respectively. d, e, Venn diagrams summarise the significantly

659 differentially expressed genes identified by the DESeq2 analyses, for each cell type, and

660 the sPLS-DA on (d) the first and (e) the second sPLS-DA component. Percentages are of

661 the total number of differentially expressed genes identified from all analyses.

662 
663 Extended Data Figure 2: Percentage of KEGG cellular processes and

664 environmental information processing (i.e. cell signalling) genes present in each

665 cell type, corresponding to the number of components making up each KEGG

666 category identified.

667 a, Cellular processes genes. b, Environmental information processing (i.e. cell

668 signalling) genes.

669

670 Extended Data Figure 3: Evolutionary age of genes expressed in Amphimedon

671 queenslandica choanocytes, archeocytes and pinacocytes using different

672 expression thresholds.

673 a-e, Phylostratigraphic enrichment of genes expressed in each cell type (Ar, archeocyte;

674 Ch, choanocyte; Pi, pinacocyte; ArCh, archeocyte + choanocyte; ArPi, archeocyte +

675 pinacocyte; ChPi, choanocyte + pinacocyte; ALL, all three cell types combined) at

676 different expression thresholds. Expressed genes are parsed into quartiles based on

677 transcript abundance in each of the cell types. Quartile 1 (Q1) includes the least

678 abundant transcripts and Q4 the most abundant. a, Phylostratigraphy enrichment of all

679 genes expressed in each of the cell types (i.e. Q1-Q4). b, Phylostratigraphy enrichment

680 of genes expressed in the top three quartiles (i.e. excluding Q1). c, Phylostratigraphy

681 enrichment of genes expressed in the top 50\% (i.e. Q3 and Q4). d, Phylostratigraphy

682 enrichment of the most highly expressed genes (i.e. Q4). e, For comparison, the

683 evolutionary age of differentially expressed genes identified using differential

684 expression analysis, DESeq2. Heat maps indicate enrichment (log odds ratio) of

685 phylostrata contained in each gene list in comparison to the A. queenslandica genome.

686 Asterisks mark significant $(\mathrm{p}<0.05)$ enrichment. The heat maps on the far right are

687 collapsed versions of the heat maps on the left, where the premetazoan category 
688 contains phylostrata from cellular to holozoan, and the poriferan category contains

689 phylostrata from poriferan to A. queenslandica. To the left of each heat map is a Venn

690 diagram, showing the number of genes in each cell type and sets of cell types. Grey

691 boxes on the heat map indicate that there were no genes in that particular gene list

692 characterised by the given phylostrata. f, Pairwise comparison illustrating the number

693 of overlapping genes for each of the quartiles between the three cell types. The numbers

694 in the cells are the number of genes common between two cell types (e.g. there are

6951569 expressed genes in common between Q2 in choanocytes and Q3 in archeocytes).

696 NE, not expressed. g, The percentage of differentially up-regulated genes identified in

697 each of the cell types using DESeq2 in the four quartiles.

698

699 Extended Data Figure 4: Orthologues shared between cell type-specific gene lists

700 and non-metazoan eukaryotes.

701 Heat map showing the percentage of A. queenslandica genes with orthogroups (OGs)

702 shared with select eukaryotes. a, Percentage of genes with OGs shared between up-

703 regulated and total expressed genes from non-exclusive lists (i.e. all genes expressed in

704 each of the three cell types, not excluding genes that overlap between any two cell

705 types). b, Percentage of genes with OGs shared between DEG and total expressed genes

706 - exclusive lists (i.e. genes uniquely up-regulated or expressed in that cell type).

708 Extended Data Figure 5: Orthologues found in Salpingoeca rosetta, Capsaspora

709 owczarzaki and Creolimax fragrantissima life cycle stages, shared with $A$.

710 queenslandica cell type transcriptomes and eukaryotic genomes.

711 a, The percent and number (in parentheses) of differentially expressed OGs found in

712 Salpingoeca rosetta, Capsaspora owczarzaki and Creolimax fragrantissima life cycle 
713 stages that are shared with Amphimedon queenslandica cell types. The numbers in

714 parentheses alongside the unicellular holozoan cell states and sponge cell type names is

715 the total number of OGs differentially expressed in that specific gene list. b, A heatmap

716 showing the percentage of OGs shared between genes differentially expressed in

717 Salpingoeca rosetta, Capsaspora owczarzaki and Creolimax fragrantissima life cycle

718 stages, and genes present in other eukaryotic genomes.

\section{Extended Data Figure 6: Heat map of transcription factor genes differentially}

expressed in choanocytes, archeocytes and pinacocytes.

72294 transcription factor genes that are differentially expressed in A. queenslandica cell

723 types are classified based on phylostratum: premetazoan (light grey); metazoan (dark

724 grey; and poriferan (black). a, Heat map of expression levels in the three cell types

725 combining all analysed CEL-Seq2 data. Gene names, families (in parentheses) and

726 phylostrata shading are shown on the right. b, Heat map of expression levels of all CEL-

727 Seq2 samples. Rows in b correspond to the rows and genes in a. c, Venn diagram

728 summary of differentially up-regulated transcription factor genes between the three cell

729 types using DESeq2. Percentages are of the total transcription factor genes differentially

730 up-regulated in all cell types. d, Bar graph of the number and distribution of

731 transcription factor genes based on evolutionary age in the three cell types.

\section{Extended Data Figure 7: Analysis of premetazoan transcription factors in}

\section{Amphimedon cells and unicellular holozoan cell states.}

735 a, The number and percentage of premetazoan transcription factor orthologues that are

736 present in the genomes of Salpingoeca rosetta, Capsaspora owczarzaki and Creolimax

737 fragrantissima. Percentages are based on the 43 premetazoan genes differentially 
738 expressed in the A. queenslandica cell types (Extended Data Fig. 5). The number of

739 transcription factor orthologues in the genome is listed above the bar. The orange bar

740 depicts the percent and number of unicellular holozoan premetazoan transcription

741 factor orthologues that are significantly differentially up-regulated in at least one cell

742 state. b, The 15 premetazoan transcription factor orthology groups (listed along the

743 top) that are significantly up-regulated in at least one Amphimedon cell type and one

744 unicellular holozoan cell state. Dots correspond to the cell types and states this occurs.

745 Black dots, orthology group with one gene member; grey dots, orthology group

746 comprised of two of more paralogues (see Supplementary File S8 for details).

748 Extended Data Figure 8: Choanocyte dedifferentiation into an archeocyte does not

749 require cell division.

750 a, b, 4 day old juveniles 6 hours after CM-DiI and EdU labelling. a, CM-DiI labelled

751 archeocytes with EdU incorporation (arrows) found near choanocyte chambers. b,

752 Labelled archeocytes without EdU incorporation (arrowheads), indicating

753 dedifferentiation from choanocytes without cell division. Scale bars: $10 \mu \mathrm{m}$.

754 c, d, Choanocyte-derived archeocytes are capable of generating new choanocyte

755 chambers. c, 4 day old juvenile 6 hours after CM-DiI and EdU labelling. Early choanocyte

756 chamber (dotted line) completely labelled with CM-DiI and EdU, indicating CM-DiI

757 labelled archeocytes, with large nuclei, are forming this chamber. The absence of cilia

758 and space at the center of this structure indicates it is not yet a functional choanocyte

759 chamber. d, 4 day old juvenile 12 hours after CM-DiI and 6 hours after EdU labelling.

760 Early choanocyte chamber (dotted line) with multiple EdU labelled cells, with both CM-

761 DiI labelled choanocytes (arrowheads) and non-CM-DiI labelled choanocytes (arrows) 
762 indicate multiple cell lineages contributing to the formation of this chamber. Scale bars:

$763 \quad$ a-d, $10 \mu \mathrm{m}$.

764

765

766

Supplementary Video Legends

767

768

Supplementary Video 1: Time-lapse video of a 4 day old juvenile Amphimedon

769

queeslandica.

770 This 10 second video captures 20 minutes of cell behavior on the outer edge of the

771 juvenile. Annotated are (i) a choanocyte chamber (cc) comprising of multiple tethered

772 choanocytes, (ii) three migrating archeocytes (ar) - there are multiple other

773 archeocytes in this video, and (iii) a pinacocyte (pi), which comes in and out of focus

774 and is characterised by a thin, transparent cytoplasm with small refractive vesicles.

775 Scale bar, $10 \mu \mathrm{m}$.

776

777 Supplementary Video 2: Capture and washing of a dissociated archeocyte.

778 All cells and choanocyte chambers used in this study were fixed or frozen in less than 15

779 minutes after dissociation from the intact sponge.

780

781

Supplementary Video 3: Time-lapse video of choanocytes transdifferentiating and

782

evacuating chambers in 4 day old juvenile Amphimedon queeslandica.

783 Matching 8-second videos captures 120 min of CM-DiI labelled choanocytes (left, red;

784 right, white), which are initially located in distinct chambers (arrows on four

785 chambers), undergoing transdifferentiation and migrating from the chambers. At the

786 end of the video, none of the CM-DiI labelled chambers are recognisable. Note that many 
787 cells vacating the choanocyte chambers are larger, consistent with choanocytes

788 dedifferentiating into larger archeocytes. Scale bar, $40 \mu \mathrm{m}$.

789

790

791

Supplementary Files

792

793

Supplementary File S1. The counting report of the cell type specific CEL-Seq2

794

samples

795

796

Supplementary File S2. Table summarising the details and the statistics of the

797

demultiplexing and mapping steps for the cell type specific CEL-Seq2 samples

798

799

Supplementary File S3. Table of differentially expressed gene lists from DESeq2

800

with BLAST2GO annotations and phylostrata ID

801

802

803

Supplementary File S4. Table of differentially expressed gene lists from sPLS-DA with BLAST2GO and KEGG annotations and phylostrata ID

804

805

Supplementary File S5. Table of KEGG enrichment analysis results on

806

differentially expressed gene lists from DESeq2

807 This spreadsheet contains the output of KEGG enrichment analyses performed on each

808 cell type DEG list. The first sheet contains percentage values of genes/components

809 identified in the DEG lists relative to the A queenslandica genome.

810 
811 Supplementary File S6. Table of the phylostrata enrichment of the differentially

812 expressed gene lists from DESeq2

813

814 Supplementary File S7. Table of cell-type gene lists and transcription factor lists

815 from the quartile expression analyses

816

817 Supplementary File S8. Table of transcription factor genes expressed in the three

818 cell types and in the differentially expressed gene lists from DESeq2 
bioRxiv preprint doi: https://doi.org/10.1101/564518; this version posted March 1, 2019. The copyright holder for this preprint (which was not certified by peer review) is the author/funder, who has granted bioRxiv a license to display the preprint in perpetuity. It is made available under a aCC-BY 4.0 International license.
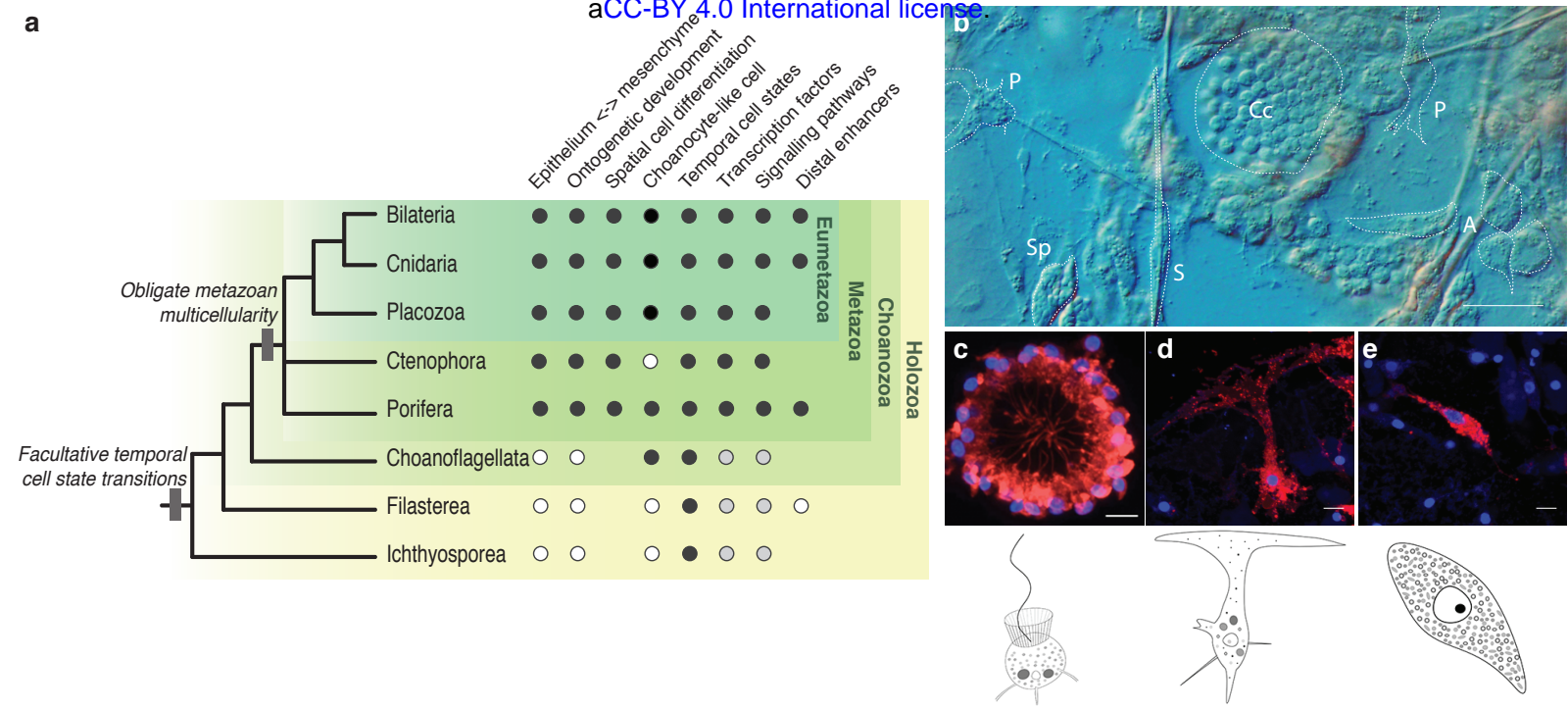

Fig. 1 
bioRyjv preprint doi: https://doi.org/10.1101/564518 cergfied by peer review) is the author/funder, who haspobanted bioRxiv a license to display the preprint Pitp perpetuity. It is made available under

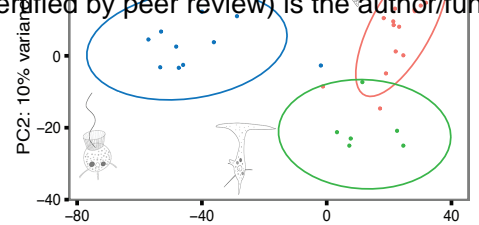

PC1: $58 \%$ variance

b

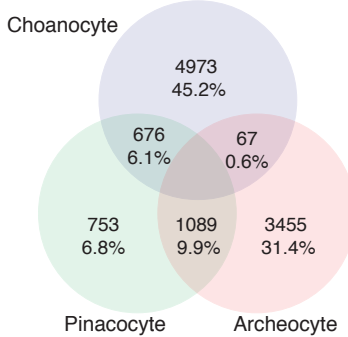

Ril6omeBY 4.0 International license.

Aminoacyl-tRNA biosynthesis

RNA transport

mRNA surveillance pathway

Ribosome biogenesis

Protein export

ER protein processing

Ubiquitin mediated proteolysis

Ubiquitin mediated prot

Proteasome

RNA degradation

DNA replication

Base excision repair

Nucleotide excision repair

Mismatch repair

Homologous recombination

Non-homologous end-joining

Fanconi anemia pathway

Translation

$\beta$-Catenin

Cadherin

$\alpha$-Cadenin

|

Percentage

$0 \quad 50 \quad 100$

Fig. 2 
bioRxiv preprint doi: https://doi.org/10.9101/564518; this versiof posted March 1, 2019. The copyright holder fol this pregint \&which was not certified by peer review) is the author/funder, who has granted bioRxiv a license to display the preprint in perpetuity. It issmagde agalable under

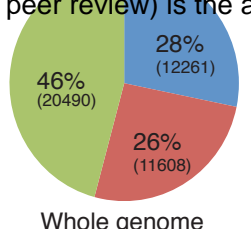

Whole genome

Sponge-specific Metazoan

- Pre-metazoan

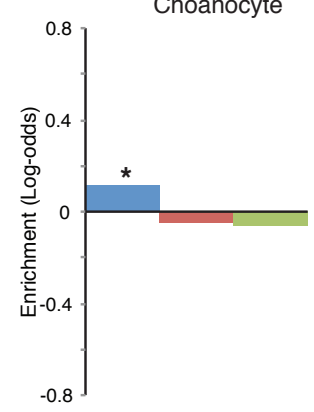

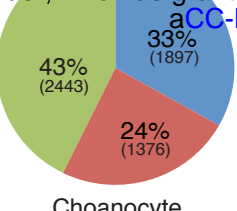
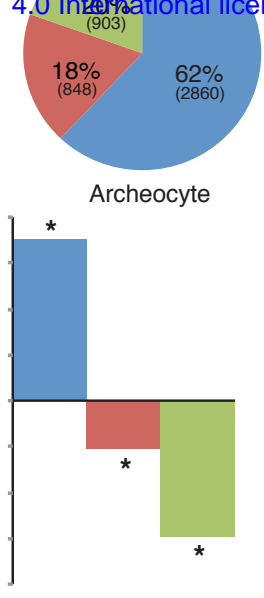
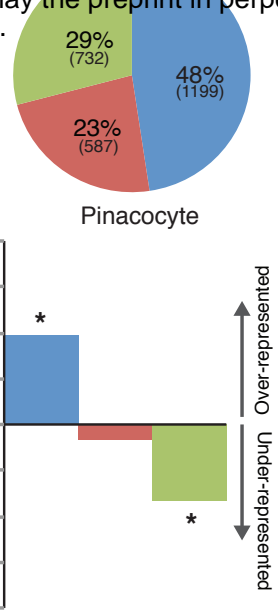

đृ.

N.s. N.s. N.s. Swimming

ஸे N.s. $5 \mathrm{e}-6$ N.s. Clonal colony

$\underset{0}{0}$ N.s. N.s. N.s. Ameoba

N.s. N.s. N.s. Aggregate colony

రำ N.s. N.s. N.s. Cyst

N.s. N.s. N.s. Ameoboid

ò N.s. 2e-22 N.s. Multinucleate

Odds ratio

Fig. 3 
bioRxiv preprint doi: https://doi.org/10.1101/564518; this version posted March 1, 2019. The copyright holder for this preprint (which was not certified by peer review) is the author/funder, who has granted bioRxiv a license to display the preprint in perpetuity. It is made available under aCC-BY 4.0 International license.

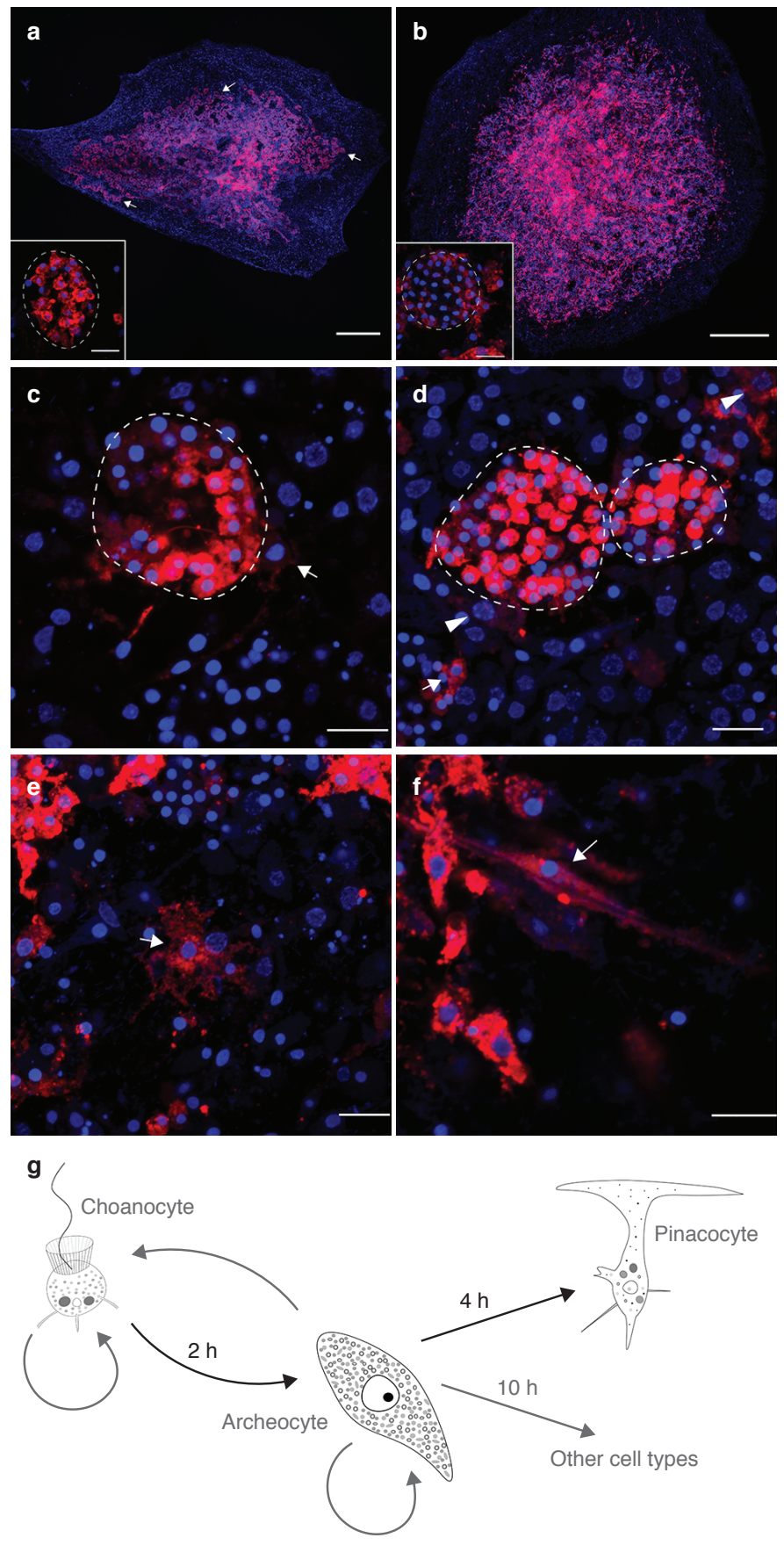

Fig. 4 


\begin{tabular}{|c|c|c|c|c|c|}
\hline Sample & Cell type & Individual & No. cells sequenced & No. reads & $\begin{array}{r}\text { Percent reads } \\
\text { mapped }\end{array}$ \\
\hline 1 & Archeocyte & $\mathrm{A}$ & 5 & 1098454 & 21.4 \\
\hline 2 & & & 5 & 10411743 & 67.6 \\
\hline 3 & & & 5 & 5699424 & 60.3 \\
\hline 4 & & & 6 & 6759553 & 72.6 \\
\hline 5 & & & 5 & 5673223 & 64.7 \\
\hline 6 & & B & 5 & 14421299 & 65.5 \\
\hline 7 & & & 5 & 9427170 & 64.0 \\
\hline 8 & & & 5 & 8208828 & 65.3 \\
\hline 9 & & & 5 & 13012311 & 71.1 \\
\hline 10 & & & 5 & 11700365 & 71.7 \\
\hline 11 & & C & 5 & 25125367 & 69.8 \\
\hline 12 & & & 5 & 15458602 & 69.7 \\
\hline 13 & & & 6 & 16070906 & 70.6 \\
\hline 14 & & & 5 & 20190551 & 71.0 \\
\hline 15 & & & 6 & 22096837 & 71.7 \\
\hline 16 & Choanocyte & A & single chamber (40-60 cells) & 9657992 & 49.2 \\
\hline 17 & & & single chamber ( $40-60$ cells) & 3864298 & 47.2 \\
\hline 18 & & & single chamber ( $40-60$ cells) & 7081396 & 59.7 \\
\hline 19 & & B & single chamber ( $40-60$ cells) & 5177297 & 61.9 \\
\hline 20 & & & single chamber ( $40-60$ cells) & 6031263 & 64.9 \\
\hline 21 & & C & single chamber ( $40-60$ cells) & 14879156 & 62.4 \\
\hline 22 & & & single chamber ( $40-60$ cells) & 12775312 & 67.0 \\
\hline 23 & & & single chamber ( $40-60$ cells) & 10569223 & 66.7 \\
\hline 24 & & & single chamber ( $40-60$ cells) & 17488774 & 64.1 \\
\hline 25 & & & single chamber ( $40-60$ cells) & 18808800 & 67.1 \\
\hline 26 & Pinacocyte & A & 5 & 19146512 & 67.6 \\
\hline 27 & & & 5 & 12081597 & 69.6 \\
\hline 28 & & & 5 & 10798371 & 67.6 \\
\hline 29 & & B & 6 & 2906098 & 58.1 \\
\hline 30 & & C & 5 & 13792427 & 60.0 \\
\hline 31 & & & 5 & 5184625 & 66.0 \\
\hline
\end{tabular}


a

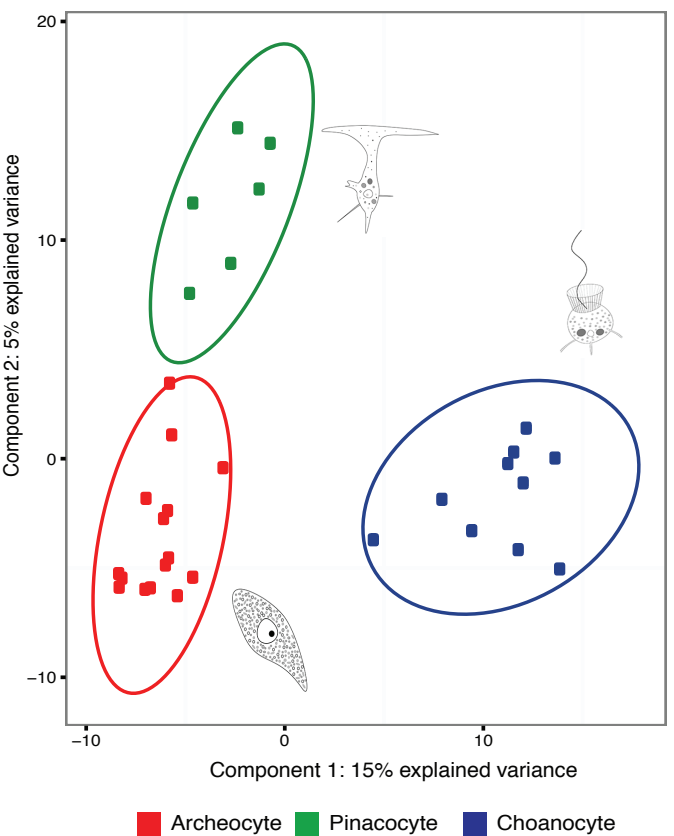

Archeocyte Pinacocyte

Choanocyte

bioRxiv preprint doi: https://doi.org/10.1101/564518; this version posted March 1, 2019. The copyright holder for this preprint (which was not certified by peer review) is the author/funder, who has granted bioRxiv a license to display the preprint in perpetuity. It is made available under

Extended Data Fig. 1

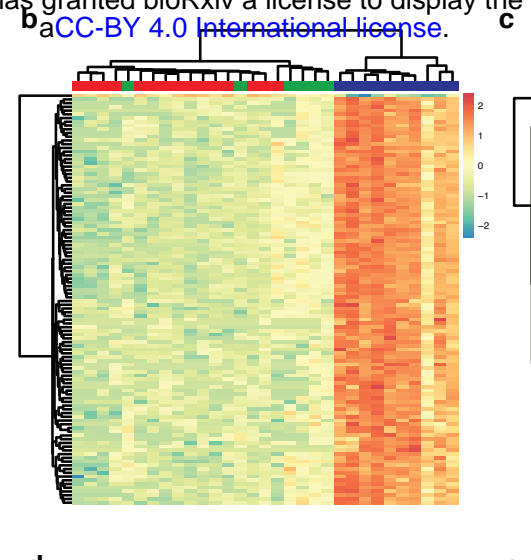

d Component DESeq2
Pinacocyte

$\underset{10}{0}$

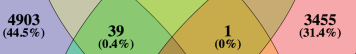

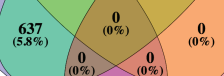

DESeq2 Choanocyte

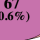

DESeq2 Archeocyte

e

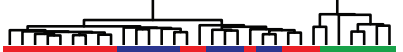

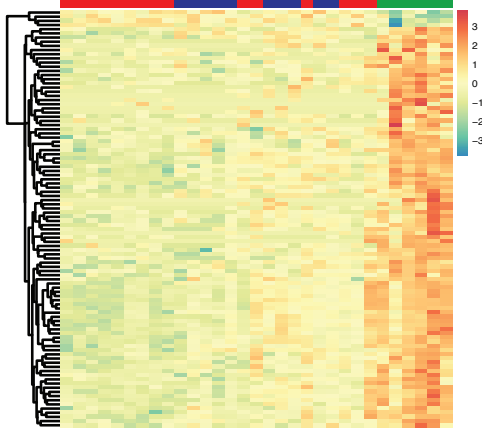

SPLS-DA DESeq2

Component $2 \quad$ Pinacocyte

$(0.1 \%)$

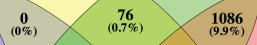

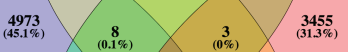

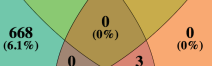

DESeq2 Choanocyte

DESeq2

Archeocyte 


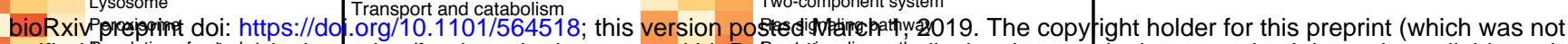

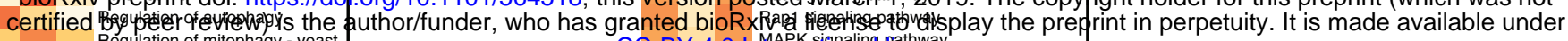
Regulation of mitophagy - yeast

Regulation of actin cytoskeleton I Cell motility

Cell cycle

Cell cycle - yeast

Cell cycle - Caulobacter

Meiosis - yeast

Oocyte meiosis

Apoptosis

Apoptosis - fly

Apoptosis - multiple species

p53 signaling pathway

Quorumsensing

Focal adhesion

Adherens junction

Tight junction

Gap junction

Signaling pathways regulating-

Cellular community

Cell growth and death

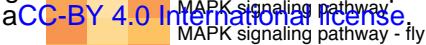

pluripotency of stem cells

Percentage

Cell growth and death

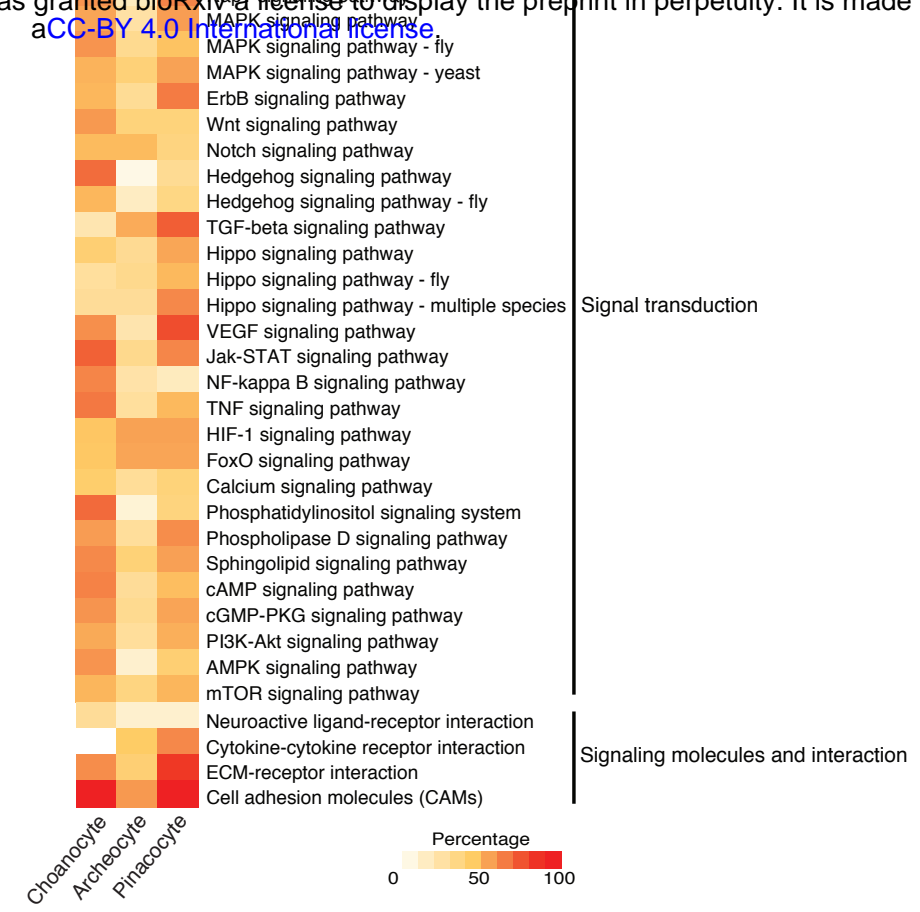

Extended Data Fig. 2 
bioRxiv preprint doi: https://doi.org/10.1101/564518; this version posted March 1, 2019. The copyright holder for this preprint (which was not certified by peer review) is the author/funder, who has granted bioRxiv a license to display the preprint in perpetuity. It is made available under ${ }^{a}$ Choanocyte

55

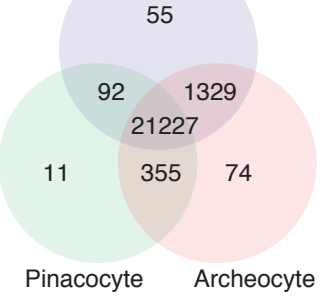

b

Choanocyte

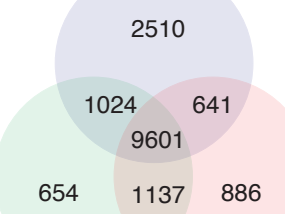

Pinacocyte Archeocyte

c
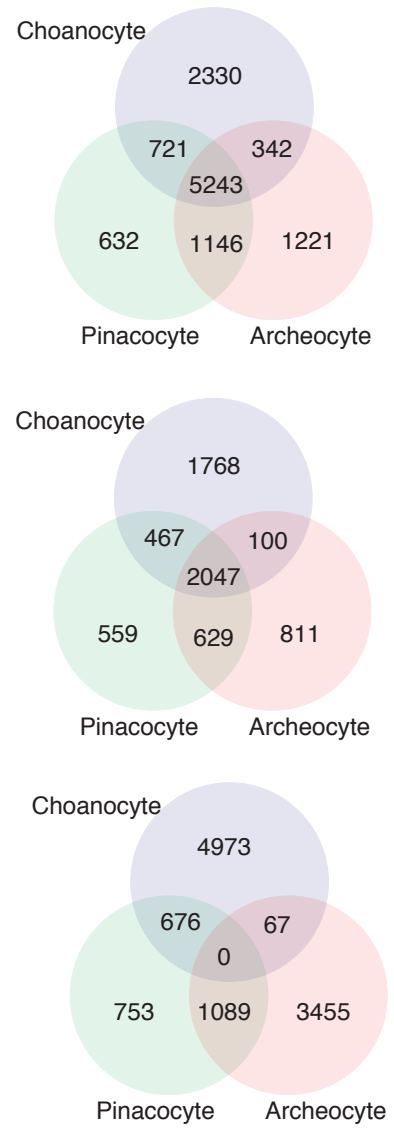
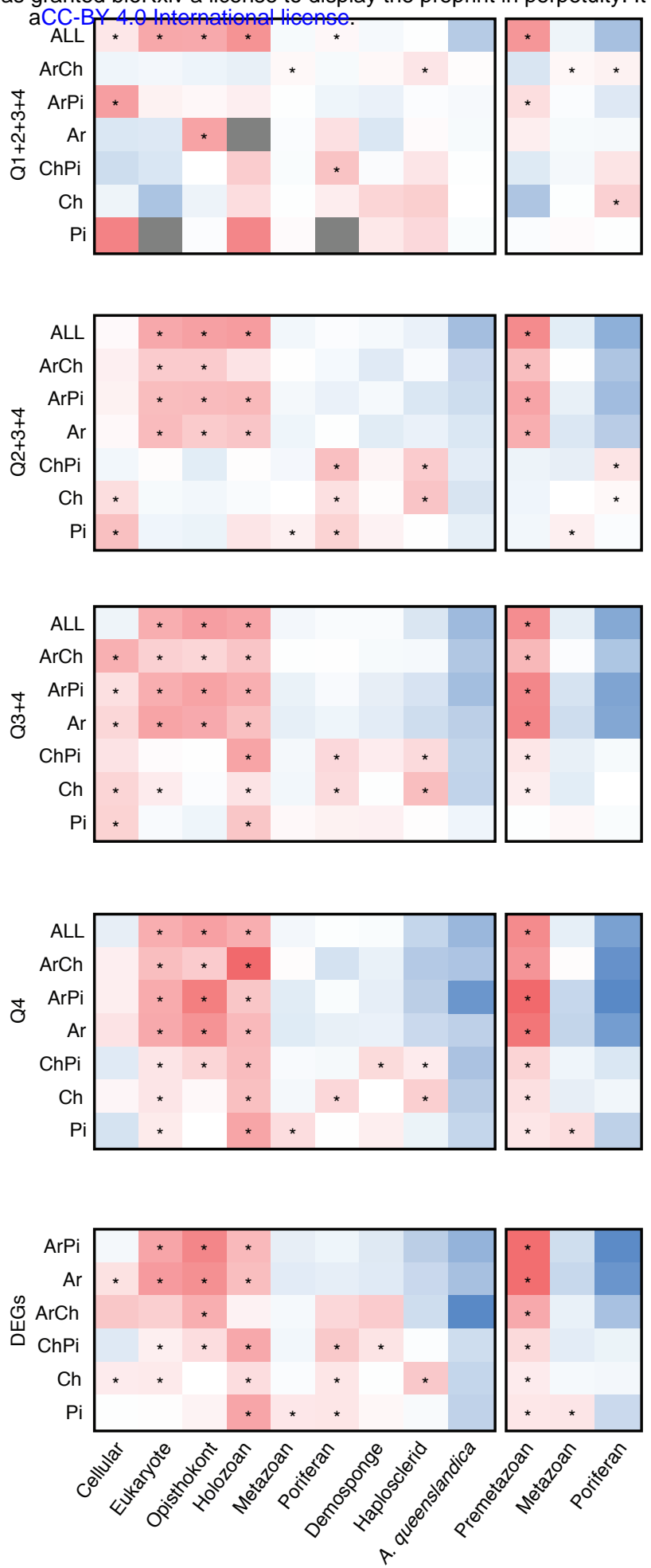

Pinacocyte

Choanocyte $\begin{array}{lllll}\text { NE } & \text { Q1 } & \text { Q2 } & \text { Q3 } & \text { Q4 }\end{array}$

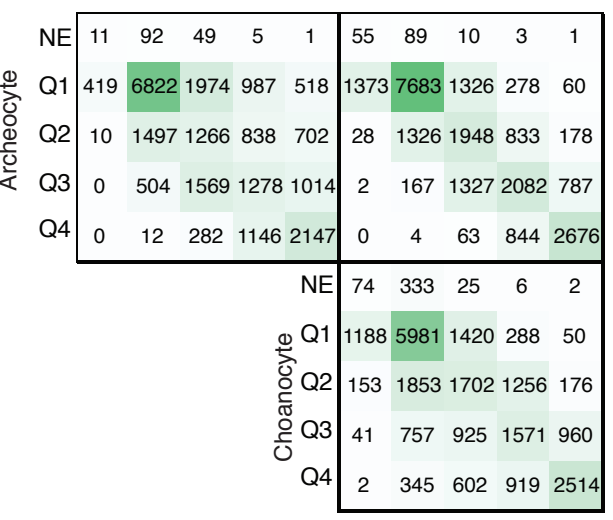

g 
bioRxiv preprint doi: https://doi.org/10.1101/564518; this version posted March 1, 2019. The copyright holder for this preprint (which was not certified by peer review) is the author/funder, who has granted bioRxiv a license to display the preprint in perpetuity. It is made available under aCC-BY 4.0 International license.

a

b

DEG (non-ex) Expressed (non-ex)

\begin{tabular}{|c|c|c|c|c|c|}
\hline $39 \%$ & $11 \%$ & $24 \%$ & $30 \%$ & $21 \%$ & $26 \%$ \\
\hline $32 \%$ & $8 \%$ & $22 \%$ & $26 \%$ & $18 \%$ & $23 \%$ \\
\hline $47 \%$ & $12 \%$ & $34 \%$ & $40 \%$ & $28 \%$ & $35 \%$ \\
\hline $45 \%$ & $17 \%$ & $33 \%$ & $39 \%$ & $30 \%$ & $36 \%$ \\
\hline $50 \%$ & $17 \%$ & $39 \%$ & $45 \%$ & $34 \%$ & $41 \%$ \\
\hline $45 \%$ & $22 \%$ & $35 \%$ & $41 \%$ & $34 \%$ & $38 \%$ \\
\hline $50 \%$ & $30 \%$ & $39 \%$ & $46 \%$ & $40 \%$ & $43 \%$ \\
\hline
\end{tabular}

DEG (exclusive)

Expressed (exclusive)

\begin{tabular}{|c|c|c|c|c|c|}
\hline $38 \%$ & $42 \%$ & $27 \%$ & $10 \%$ & $14 \%$ & $8 \%$ \\
\hline $31 \%$ & $37 \%$ & $21 \%$ & $7 \%$ & $11 \%$ & $10 \%$ \\
\hline $5 \%$ & $52 \%$ & $29 \%$ & $11 \%$ & $22 \%$ & $18 \%$ \\
\hline $1 \%$ & $51 \%$ & $27 \%$ & $16 \%$ & $22 \%$ & $19 \%$ \\
\hline$\%$ & $56 \%$ & $33 \%$ & $15 \%$ & $30 \%$ & $22 \%$ \\
\hline $1 \%$ & $49 \%$ & $32 \%$ & $21 \%$ & $26 \%$ & $22 \%$ \\
\hline ०० & $54 \%$ & $39 \%$ & $29 \%$ & $33 \%$ & $24 \%$ \\
\hline
\end{tabular}

\begin{tabular}{|c|c|c|c|c|c|c|}
\hline $29 \%$ & $34 \%$ & $34 \%$ & $20 \%$ & $8 \%$ & $9 \%$ & $10 \%$ \\
\hline & $27 \%$ & $26 \%$ & $15 \%$ & $5 \%$ & $7 \%$ & $8 \%$ \\
\hline $0 \%$ & $42 \%$ & $41 \%$ & $23 \%$ & $7 \%$ & $12 \%$ & $12 \%$ \\
\hline p\% & $41 \%$ & $40 \%$ & $27 \%$ & $13 \%$ & $18 \%$ & $13 \%$ \\
\hline & $45 \%$ & $45 \%$ & $31 \%$ & $11 \%$ & $20 \%$ & $14 \%$ \\
\hline$\%$ & $39 \%$ & $40 \%$ & $33 \%$ & $19 \%$ & $22 \%$ & $16 \%$ \\
\hline $7 \%$ & $45 \%$ & $44 \%$ & $36 \%$ & $29 \%$ & $29 \%$ & $18^{\circ}$ \\
\hline
\end{tabular}

Arabidopsis thaliana

Saccharomyces cerevisiae

Creolimax fragrantissima

Sphaeroforma arctica

Capsaspora owczarzaki

Monosiga brevicollis

Salpingoeca rosetta

50

$40 \underset{\varnothing}{7}$

$30 \stackrel{\mathbb{D}}{=}$

Extended Data Fig. 4 
bioRxiv preprint doi: https://doi.org/10.1101/564518; this version posted March 1, 2019. The copyright holder for this preprint (which was not certified by peer review) is the author/funder, who has granted bioRxiv a license to display the preprint in perpetuity. It is made available under

a

\begin{tabular}{r|ccc|}
\multicolumn{3}{c}{ Salpingoeca rosetta } \\
\cline { 2 - 4 } Sessile (503) & $10 \%$ & $16 \%$ & $4 \%$ \\
& $(52)$ & $(80)$ & $(18)$ \\
Swimming (316) & $16 \%$ & $16 \%$ & $4 \%$ \\
& $(52)$ & $(50)$ & $(12)$ \\
Clonal colony (131) & $4 \%$ & $31 \%$ & $1 \%$ \\
& $(5)$ & $(41)$ & $(1)$ \\
\cline { 2 - 4 } & & &
\end{tabular}

Capsaspora owczarzaki

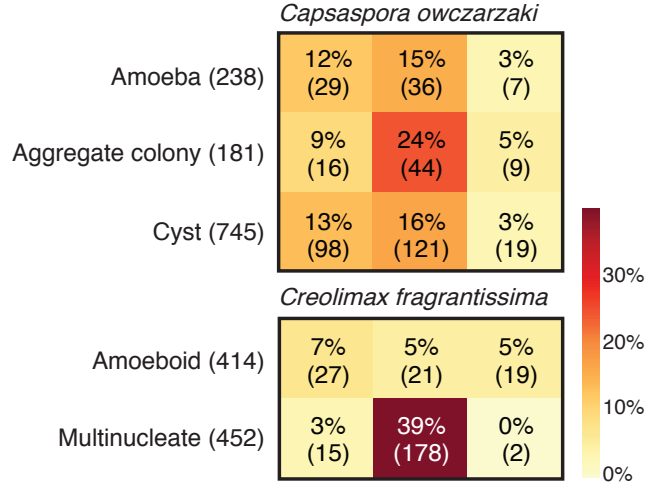

b
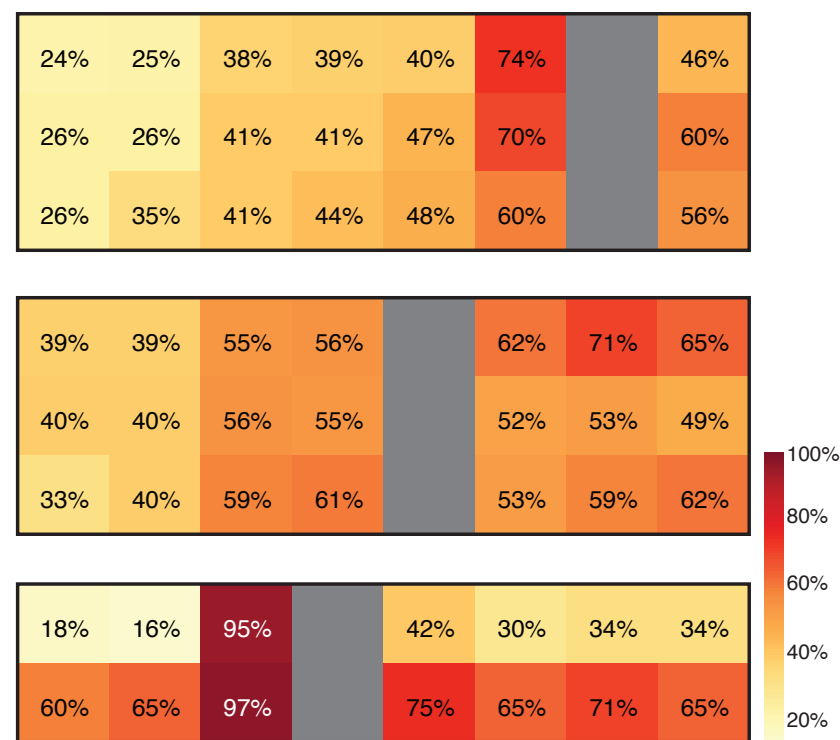

\section{Extended Data Fig. 5}


bioRxiv preprint AREX)(RFX)

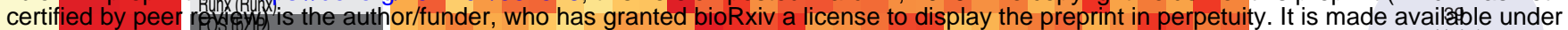

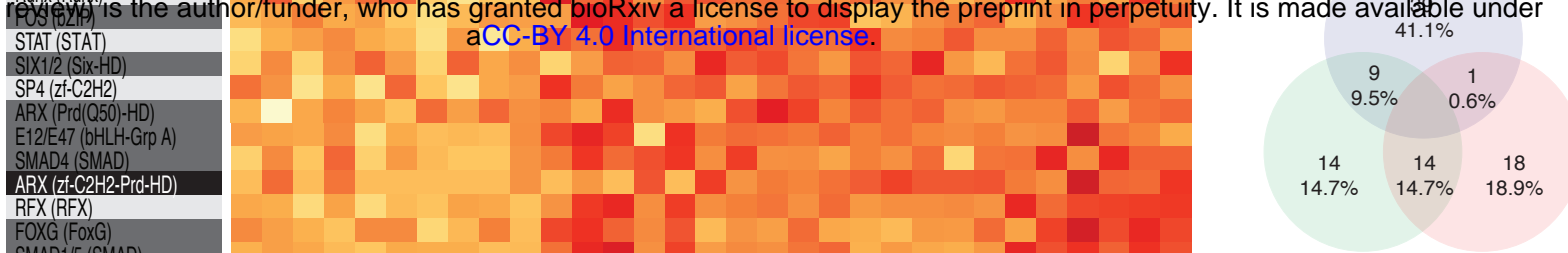

Pinacocyte Archeocyte

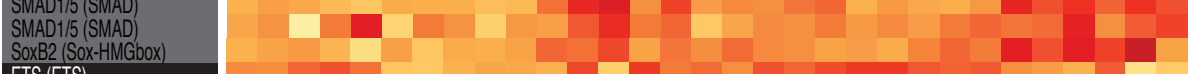

ETS (ETS)

VPS72 (VPST2YL1)

KUX1/2 (Cut-HD)

HMGB1 (HMGbox)

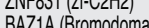

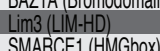

$\operatorname{Max}(b+H-G r \operatorname{Br})$

Myc (bHLH-Grp B)

Myc (bH
GATA

GATA
E2F7 (E2F)

E2F7 (E2F)
CP2 (CP2)

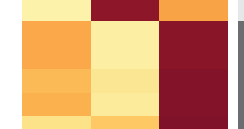

Islet (LIM-HD)
NFIA (CTFNFI)

NFIA (CTFNF1)
Bsh (Anto-HD)

RX (Prd( (O50)-HD)

Hifisim/Th (bHLH-Grp C)

EGR1 (EGR Zt-C2H2)

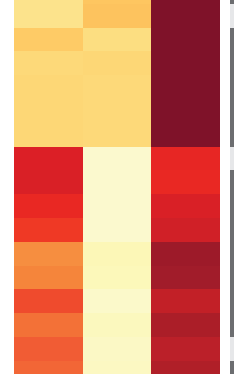

FoxP (FoxP)

KLF1/2/4 (KLF/SP)

Glass-like (tz-COH?)

FoxL2 (FoxL2)

CLOCK (bHLH-Grp C)

FoxL2 (FoxL2)

Notch (Notch
IREA (IRF)

Tbx4/5 (T-box 4/5)

KLF $/ 13$ (KLF/SP)

ARID3 (ARID)

IRF4 (IRF)
PAR-L (bZIP.PAR)

YY1 (Zt-C2H2)

Hey (bHLH-Grpe)

ATr-2(DZZP-A

MTF (bHHH-Gro B)

MITF (bHLH-Grp B)
KLF6/7 (KLFISP)

FoxJ2/3(FoxJ2/3)

ARNT-ike (bHLH-Grp C)

NR2 (Nucle)

FoxN2/3 (FoxN2/3)
FoxO (FoxO)
NEE2h (h7IP.CNC)

CSL (CSL)

FoxK (FoxK)

SoxC (SOx-HMGb
FoxJ1 (FoxJ1)

AP4 (bHLH-Grp B

ATF4 (bZIP-ATF4/5)

PARB (b-ZZIP-PAR)

XBP1 (DZIP-XBP)

FoxO-ike (FoxO)

ZNF410 (Zt-C2H2)

GRHL1 (GRH-CP2)

NFYC (NFYCIHAP5)

NF-KB (RHD)

CREBBP (CPB//300)

Tbx1/15/20 (T-boxt/15/20)
GLL/2/ (alilalis/nk//2ic)

SREBP (bHLH-Grp B

MAF (LZZP-MAF)
NK5/67 (Antp-HD)

CEBP (bZZPP.CEBP)

COE (bHLH-Grr F)

KDM5 (Zt-C2H2)
Tub (TULP)

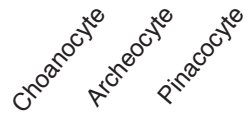

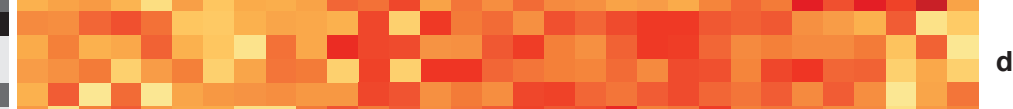

d

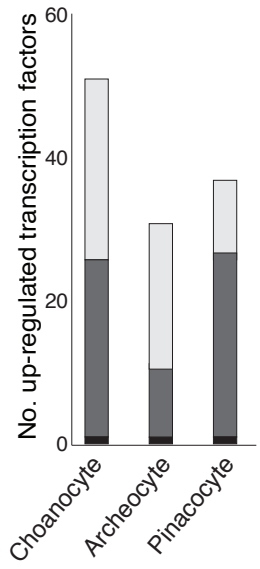

$\square$ Premetazoan origin

$\square$ Metazoan origin

- Poriferan origin

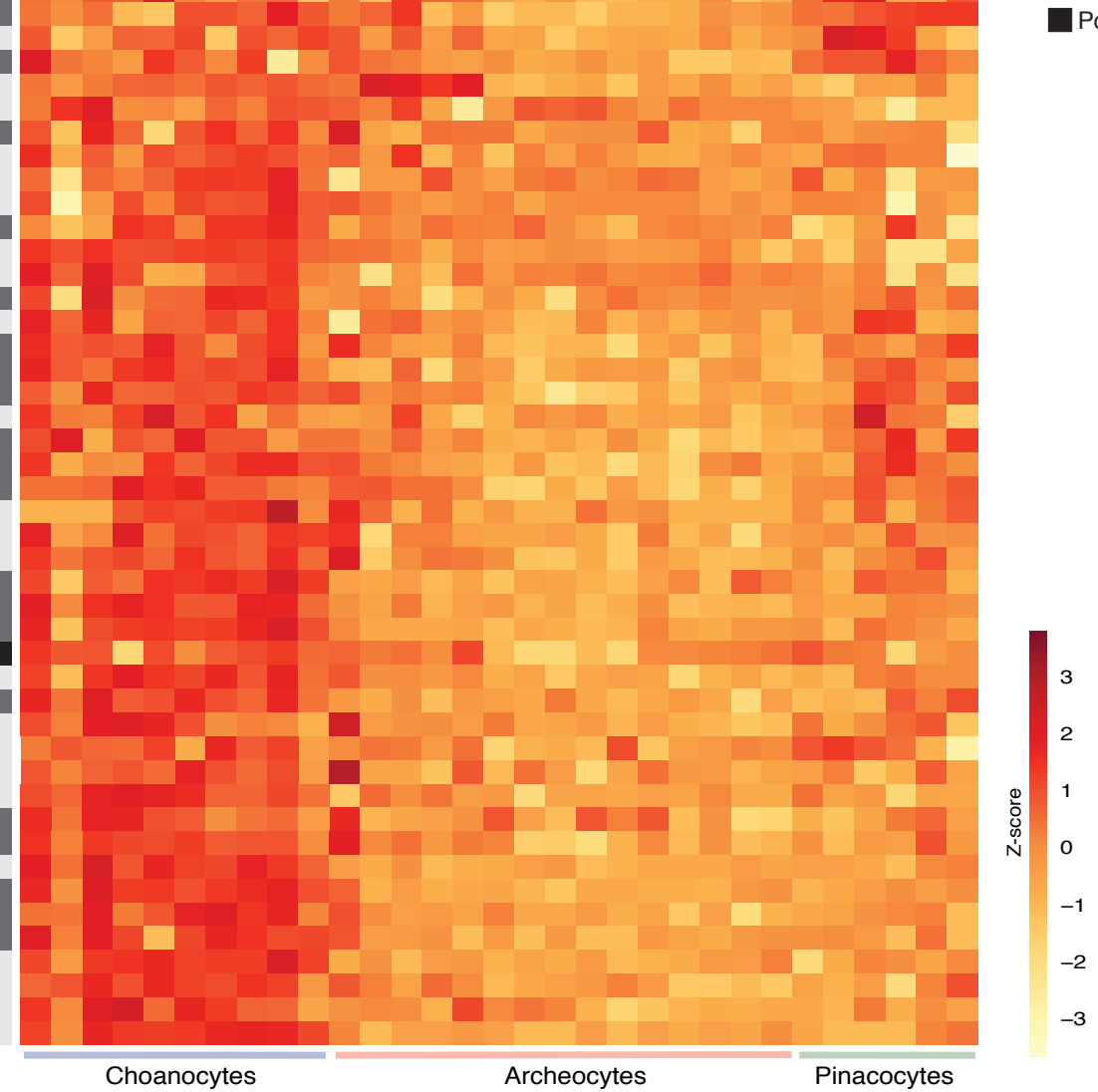

Extended Data Fig. 6 
bioRxiv preprint doi: https://doi.org/10.1101/564518; this version posted March 1, 2019. The copyright holder for this preprint (which was not certified by peer review) is the author/funder, who has granted bioRxiv a license to display the preprint in perpetuity. It is made available under

a

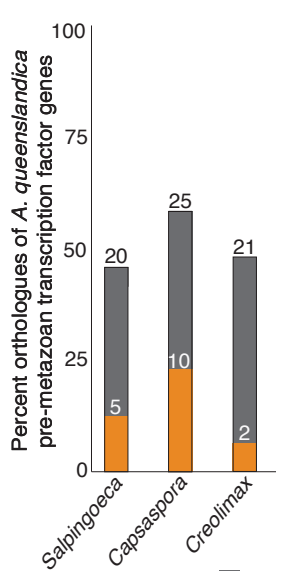

b aCC-BY 4.0 International license.

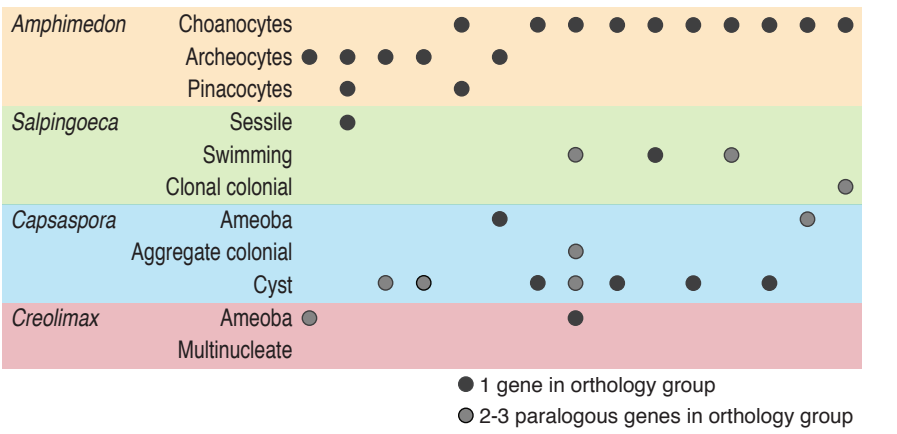

Orthology groups present in the genome (number above)

Orthology groups differentially expressed (number above)

\section{Extended Data Fig. 7}


bioRxiv preprint doi: https://doi.org/10.1101/564518; this version posted March 1, 2019. The copyright holder for this preprint (which was not certified by peer review) is the author/funder, who has granted bioRxiv a license to display the preprint in perpetuity. It is made available under aCC-BY 4.0 International license.

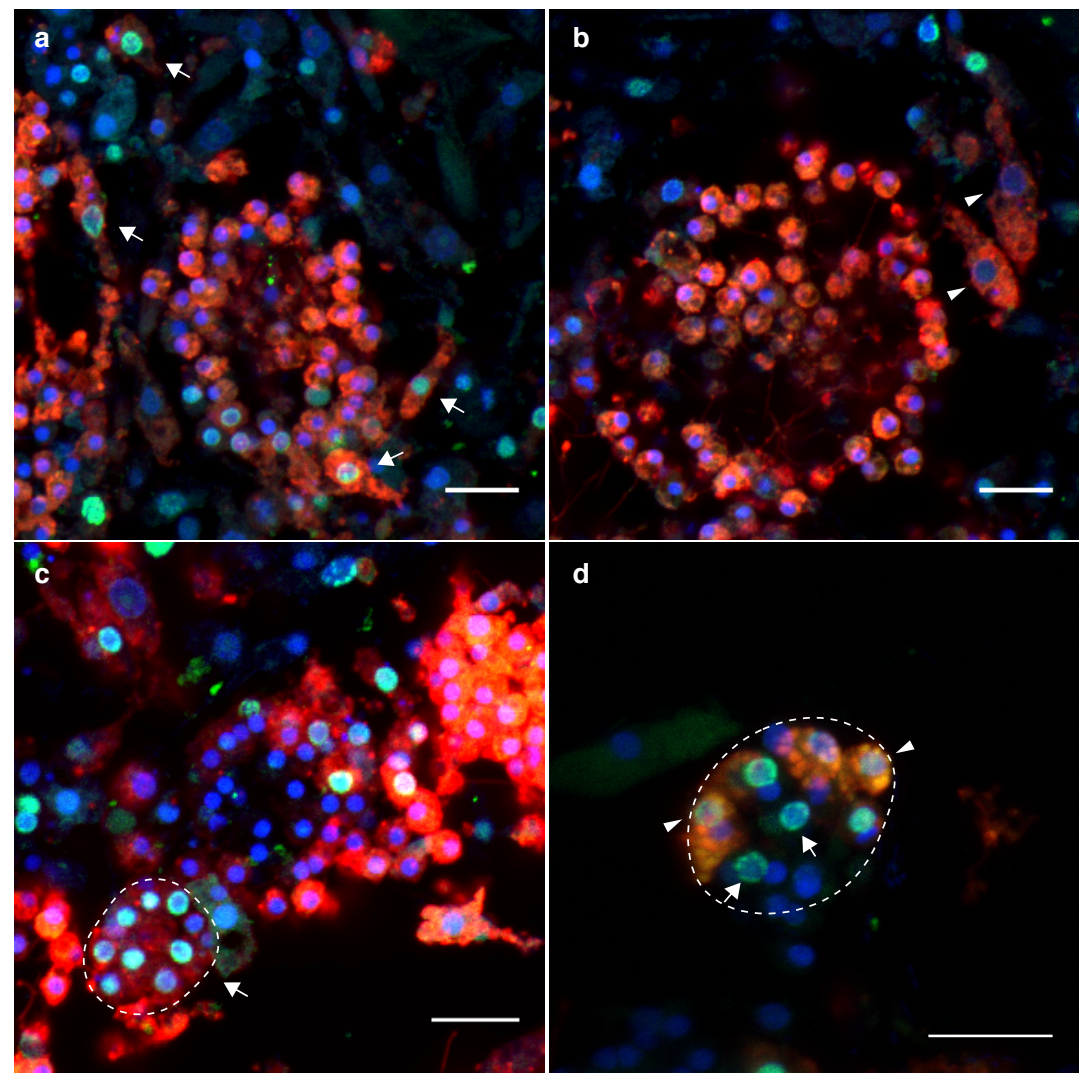

\section{Extended Data Fig. 8}

